\title{
SUPPLEMENTAL FIGURES \\ for \\ New Peak Detection Performance Metrics from \\ the MAM Consortium Interlaboratory Study
}

Trina Mouchahoir ${ }^{1,2, *}$, John E. Schiel ${ }^{1,2}$, Rich Rogers ${ }^{3}$, Alan Heckert ${ }^{1}$, Benjamin J. Place ${ }^{1}$, Aaron Ammerman ${ }^{4}$, Xiaoxiao $\mathrm{Li}^{4}$, Tom Robinson ${ }^{4}$, Brian Schmidt ${ }^{4}$, Chris M. Chumsae ${ }^{5}$, Xinbi $\mathrm{Li}^{5}$, Anton V. Manuilov ${ }^{5}$, Bo Yan ${ }^{5}$, Gregory O. Staples ${ }^{6}$, Da Ren ${ }^{7}$, Alexander J. Veach ${ }^{7}$, Dongdong Wang $^{8}$, Wael Yared ${ }^{8}$, Zoran Sosic ${ }^{9}$, Yan Wang ${ }^{9}$, Li Zang ${ }^{9}$, Anthony M. Leone ${ }^{10}$, Peiran Liu ${ }^{10}$, Richard Ludwig ${ }^{10}$, Li Tao ${ }^{10}$, Wei Wu ${ }^{10}$, Ahmet Cansizoglu ${ }^{11}$, Andrew Hanneman ${ }^{11}$, Greg W. Adams $^{12}$, Irina Perdivara ${ }^{12}$, Hunter Walker ${ }^{12}$, Margo Wilson ${ }^{12}$, Arnd Brandenburg ${ }^{13}$, Nick DeGraan-Weber ${ }^{14}$, Stefano Gotta ${ }^{13}$, Joe Shambaugh ${ }^{14}$, Melissa Alvarez ${ }^{15}$, X. Christopher Yu ${ }^{15}$, Li $\mathrm{Cao}^{16}$, Chun Shao ${ }^{16}$, Andrew Mahan ${ }^{17}$, Hirsh Nanda ${ }^{17}$, Kristen Nields ${ }^{17}$, Nancy Nightlinger ${ }^{3}$, Helena Maria Barysz ${ }^{18}$, Michael Jahn ${ }^{18}$, Ben Niu ${ }^{19}$, Jihong Wang ${ }^{19}$, Gabriella Leo ${ }^{20}$, Nunzio Sepe $^{20}$, Yan-Hui Liu ${ }^{21}$, Bhumit A. Patel ${ }^{21}$, Douglas Richardson ${ }^{21}$, Yi Wang ${ }^{21}$, Daniela Tizabi ${ }^{1,2}$, Oleg V. Borisov ${ }^{22}$, Yali Lu ${ }^{22}$, Ernest L. Maynard ${ }^{22}$, Albrecht Gruhler ${ }^{23}$, Kim F. Haselmann ${ }^{23}$, Thomas N. Krogh ${ }^{23}$, Carsten P. Sönksen ${ }^{23}$, Simon Letarte ${ }^{24}$, Sean Shen ${ }^{24}$, Kristin Boggio ${ }^{25}$, Keith Johnson ${ }^{25}$, Wenqin $\mathrm{Ni}^{25}$, Himakshi Patel ${ }^{25}$, David Ripley ${ }^{25}$, Jason C. Rouse ${ }^{25}$, Ying Zhang ${ }^{25}$, Carly Daniels $^{26}$, Andrew Dawdy ${ }^{26}$, Olga Friese ${ }^{26}$, Thomas W. Powers ${ }^{26}$, Justin B. Sperry ${ }^{26}$, Josh Woods $^{26}$, Eric Carlson ${ }^{27}$, K. Ilker Sen ${ }^{27}$, St John Skilton ${ }^{27}$, Michelle Busch ${ }^{28}$, Anders Lund ${ }^{28}$, Martha Stapels ${ }^{28}$, Xu Guo ${ }^{29}$, Sibylle Heidelberger ${ }^{29}$, Harini Kaluarachchi ${ }^{29}$, Sean McCarthy ${ }^{30}$, John $\mathrm{Kim}^{31}$, Jing Zhen ${ }^{31}$, Ying Zhou ${ }^{31}$, Sarah Rogstad $^{32}$, Xiaoshi Wang ${ }^{32}$, Jing Fang ${ }^{33}$, Weibin Chen $^{33}$, Ying Qing Yu ${ }^{33}$, John G. Hoogerheide ${ }^{34}$, Rebecca Scott ${ }^{34}$ and Hua Yuan ${ }^{34}$

${ }^{1}$ National Institute of Standards and Technology, 100 Bureau Dr, Gaithersburg, MD, 20899, U.S.A.

${ }^{2}$ Institute for Bioscience and Biotechnology Research, 9600 Gudelsky Dr, Rockville, MD, 20850, U.S.A.

${ }^{3}$ Just - Evotech Biologics, 401 Terry Ave N., Seattle, WA, 98109, U.S.A.

${ }^{4}$ AbbVie, 1500 Seaport Blvd, Redwood City, CA, 94063, U.S.A.

${ }^{5}$ AbbVie, 100 Research Drive, Worcester, MA, 01605, U.S.A.

${ }^{6}$ Agilent Technologies, 5301 Stevens Creek Blvd, Santa Clara, CA, 95008, U.S.A.

${ }^{7}$ Amgen, One Amgen Center Dr, Thousand Oaks, CA, 91320, U.S.A.

${ }^{8}$ BioAnalytix, 790 Memorial Dr, Cambridge, MA, 02139, U.S.A.

${ }^{9}$ Biogen, 125 Broadway, Cambridge, MA, 02142, U.S.A.

${ }^{10}$ Bristol-Myers Squibb, 311 Pennington-Rocky Hill Road, Pennington, NJ, 08534, U.S.A.

${ }^{11}$ Charles River Laboratories, 8 Henshaw Street, Shrewsbury, MA, 01801, U.S.A.

${ }^{12}$ FUJIFILM Diosynth Biotechnologies, 101 J. Morris Commons Ln, Morrisville, NC, 27560, U.S.A.

${ }^{13}$ Genedata, Margarethenstrasse 38, Basel, 4053, Switzerland

${ }^{14}$ Genedata, 750 Marrett Road, One Cranberry Hill, Lexington, MA, 02421, U.S.A.

${ }^{15}$ Genentech, 1 DNA Way, South San Francisco, CA, 94080, U.S.A.

${ }^{16}$ GSK, 709 Swedeland Rd, King of Prussia, PA, 19406, U.S.A.

${ }^{17}$ Janssen, 1400 McKean Road, Springhouse, PA, 19477, U.S.A.

${ }^{18}$ Lonza, Hochbergerstrasse 60 A, Basel, 4057, Switzerland 
${ }^{19}$ AstraZeneca, One MedImmune Way, Gaithersburg, MD, 20878, U.S.A.

${ }^{20}$ EMD Serono, Via Luigi Einaudi 11, Guidonia Montecelio (Roma), 00012, Italy; an affiliate of Merck KGaA, Darmstadt, Germany

${ }^{21}$ Merck \& Co., Inc., 2000 Galloping Hill Rd, Kenilworth, NJ, 07033, U.S.A.

${ }^{22}$ Novavax, Inc., 20 Firstfield Road, Gaithersburg, MD, 20878, U.S.A.

${ }^{23}$ Novo Nordisk, Novo Nordisk Park, Måløv, 2760, Denmark

${ }^{24}$ Pfizer, 375 N Field Dr, Lake Forest, IL, 60045, U.S.A.

${ }^{25}$ Pfizer, 1 Burtt Rd, Andover, MA, 01810, U.S.A.

${ }^{26}$ Pfizer, 700 Chesterfield Pkwy West, Chesterfield, MO, 63017, U.S.A.

${ }^{27}$ Protein Metrics Inc, 20863 Stevens Creek Blvd, Cupertino, CA, 95014, U.S.A.

${ }^{28}$ Sanofi, 1 The Mountain Rd, Framingham, MA, 01701, U.S.A.

${ }^{29}$ SCIEX, 71 Four Valley Drive, Concord, ON, L4K 4V8, Canada

${ }^{30}$ SCIEX, 500 Old Connecticut Path, Framingham, MA, 01701, U.S.A.

${ }^{31}$ Teva, 145 Brandywine Pkwy, West Chester, PA, 19380, U.S.A.

${ }^{32}$ U.S. Food and Drug Administration, 10903 New Hampshire Ave, Silver Spring, MD, 20993, U.S.A.

${ }^{33}$ Waters, 34 Maple St, Milford, MA, 01757, U.S.A.

${ }^{34}$ Zoetis, 333 Portage St, Kalamazoo, MI, 49007, U.S.A.

*Corresponding author: trina.mouchahoir@nist.gov; (240) 314-6217

Disclaimer. Certain commercial equipment, instruments, or materials are identified in this paper in order to specify the experimental procedure adequately. Such identification is not intended to imply recommendation or endorsement by the National Institute of Standards and Technology, nor is it intended to imply that the materials or equipment identified are necessarily the best available for the purpose. NISTmAb values and information reported herein should not be construed as certified values and do not replace or supersede those presented in any Report of Investigation (ROI) for the Reference Material. NISTmAb users should always refer to the ROI associated with their specific material lot for the most up to date values and uncertainty ranges (https://www-s.nist.gov/srmors/view_detail.cfm?srm=8671). 


\section{Table of Contents}

Figure S1. Preparation of pH Stress, Reference, Unknown and Spike Samples

Full Caption

a) degradation of NISTmAb under high $\mathrm{pH}$ stress conditions _.....................................

b) tryptic digestion of $\mathrm{pH}$ stressed NISTmAb and unstressed NISTmAb _....................S3

Figure S2. Survey of Instrument and Software Types Used for Data Acquisition and Analysis

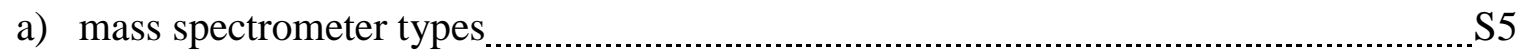

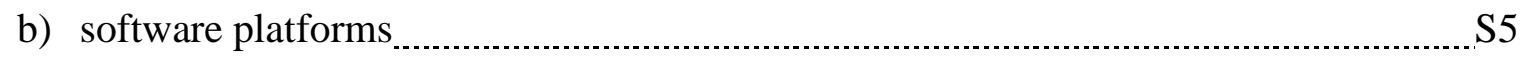

Figure S3. Intralaboratory Standard Deviations of Calibration Sample Peptide Retention Times...... S6

Figure S4. Intralaboratory Calibration Sample Peptide Mass Accuracy........................................... S7

Figure S5. Intralaboratory Fold-Change Values of Calibration Sample Peptides __......................... S8

Figure S6. Distribution of FCD Thresholds Applied _........................................................... S9

Figure S7. Distribution of NPD Thresholds Applied

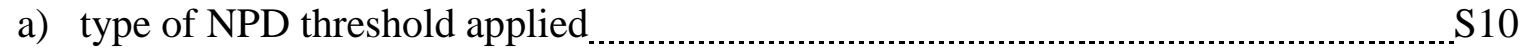

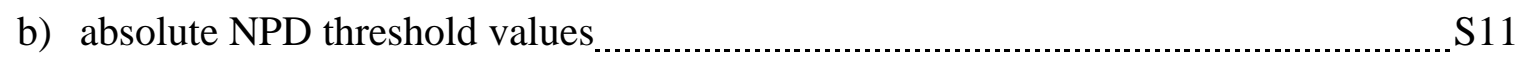

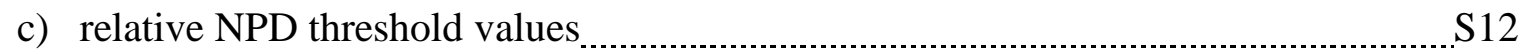

Figure S8. In-Source Fragment Spectra Generated by Spike Peptide DIPVPKPK __................... S13

Figure S9. Tally of Participants Whose Results Conformed to Expectation for Spike and Unknown

NPD Analyses

S14

Figure S10. Instrument Performance Relative to Conformity Status

a) retention time standard deviation $\ldots$

b) $|\mathrm{ppm}| \ldots \ldots$

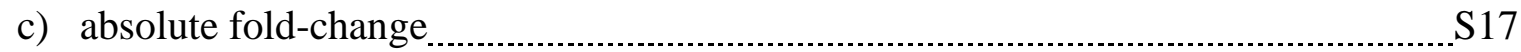

d) absolute fold-change standard deviation ___ $\quad$ S18

Figure S11. NPD Thresholds Relative to Conformity Status

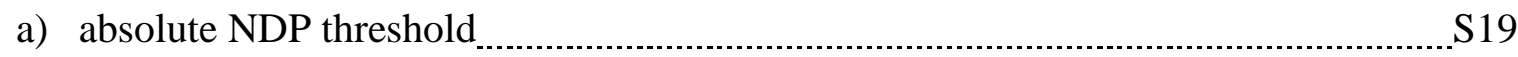

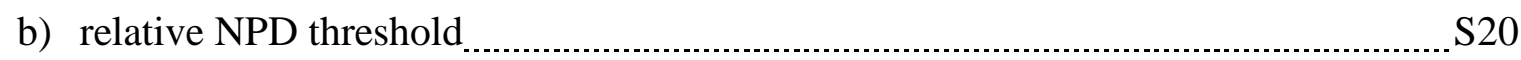

Figure S12. FCD Thresholds Relative to Conformity Status _................................................... S21

Figure S13. Survey of Modifications Reported in pH Stress Sample _........................................... S22

Figure S14. Number of Peaks Reported in pH Stress Sample Compared to Absolute NPD threshold Applied.

Figure S15. Potential Methods for Determining FCD Threshold

a) reproducibility standard deviation $\quad$ S24

b) upper confidence limit _ _ _ _

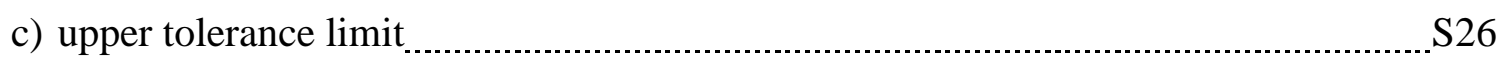

Figure S16. Summary of Box Plot Interpretation _....................................................... S27

Figure S17. Extracted Ion Chromatograms of PENNY Peptide Deamidation Species _.................... S28 
Figure S1. Preparation of pH Stress, Reference, Unknown and Spike Samples. Schematic representation of the $\mathrm{pH}$ Stress [(a) Step 1 through Step 4] and tryptic digestion protocols [(b) Step 5 through Step 18] used to prepare the Reference and Test Samples for the interlaboratory round robin study. For all samples, the digestion protocol began immediately after completion of the $\mathrm{pH}$ Stress protocol. Note that the Reference, Unknown, and Spike Samples were the product of the same digestion, performed in parallel with the $\mathrm{pH}$ Stress Sample. Vials labeled as "Bulk" refer to the sample which is destined to be split after digestion into the samples known as Reference, Unknown, and Spike. In Step 16, the pH, Reference and Unknown vials were diluted to 0.25 $\mu \mathrm{g} / \mu \mathrm{L}$ with $0.1 \%$ FA as noted, but dilution of the Spike Sample to $0.25 \mu \mathrm{g} / \mu \mathrm{L}$ was actually achieved through a combination of Steps 16 and 17. The Spike Peptides added to this sample were resuspended in $0.1 \%$ FA. The volume of $0.1 \%$ FA added during Step 16 plus the volume of 0.1 $\%$ FA from the Spike Peptides in Step 17 together brought the final protein concentration to 0.25 $\mu \mathrm{g} / \mu \mathrm{L}$. In addition to the samples detailed here, one "blank" was prepared in parallel with the $\mathrm{pH}$ vial (from Step 1 through Step 12) and another "blank" was prepared in parallel with the Bulk vial (from Step 5 through Step 12) in order to provide an accurate background subtraction when measuring protein concentrations. Each blank was processed using the same steps detailed for its NISTmAb counterpart, except that formulation buffer was transferred to the vial in the initial step instead of $10 \mu \mathrm{g} / \mu \mathrm{L}$ NISTmAb. Details regarding the composition of buffers are given within the text of the Materials and Methods section of the manuscript. DTT = dithiothreitol; IAM = iodoacetamide; Red = reduced; $\mathrm{R} \& \mathrm{~A}=$ reduced and alkylated; dig = digest; $\mathrm{RT}=$ room temperature; $\mathrm{FA}=$ formic acid.

Figure S1 (a and b) are found on the following three pages. 
Figure S1 (continued). Preparation of pH Stress, Reference, Unknown and Spike Samples.

a) Degradation of NISTmAb under high pH stress conditions.

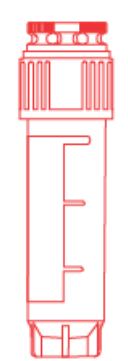

$10 \mu \mathrm{g} / \mu \mathrm{L}$ NISTmAb in Formulation Buffer
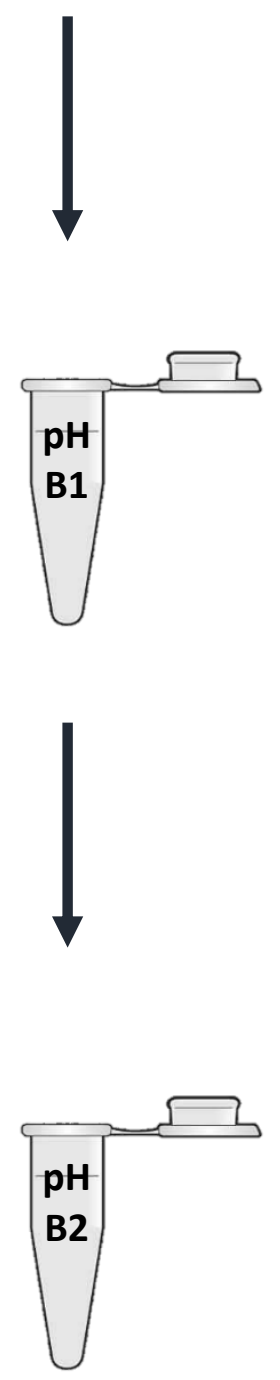

(1)

Exchange to $\mathrm{pH}$ Stress Buffer (B1)

(2)

Incubate at $40{ }^{\circ} \mathrm{C}, 8$ days

(3)

Exchange to $10.1 \%$ Formulation Buffer/

$89.9 \%$ Denaturing Buffer (B2)

(4)

Measure protein concentration of $\mathrm{pH}$ B2 vial contents 
Figure S1 (continued). Preparation of pH Stress, Reference, Unknown and Spike Samples.

b) Tryptic digestion of pH stressed NISTmAb and unstressed NISTmAb.

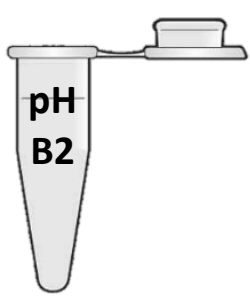

$x \mu \mathrm{g} / \mu \mathrm{L} \mathrm{pH}$ stressed NISTmAb (from Step 4)

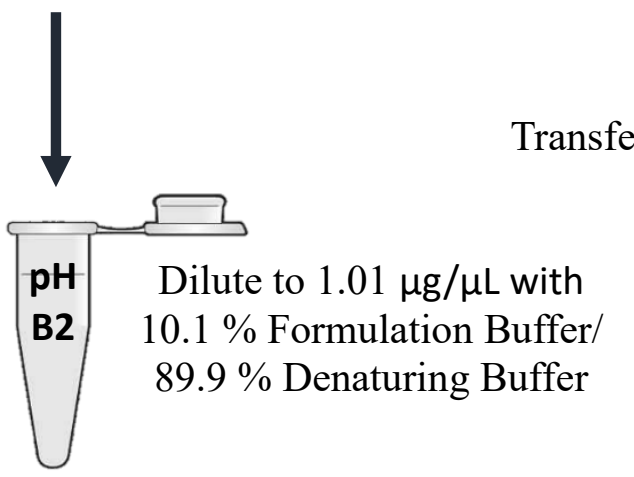

(5)

er $x \mu \mathrm{g}$ protein

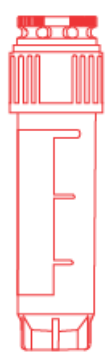

$10 \mu \mathrm{g} / \mu \mathrm{L}$ NISTmAb in Formulation Buffer

(6)
Dilute to $1.01 \mu \mathrm{g} / \mu \mathrm{L}$ with Denaturing Buffer

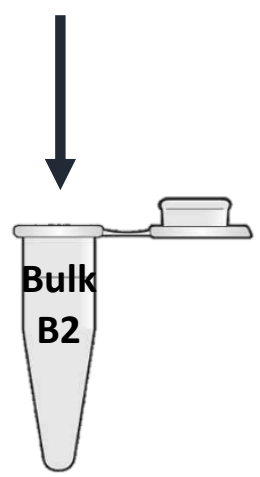

Buffer composition in both vials is now $10.1 \%$ Formulation, $89.9 \%$ Denaturing
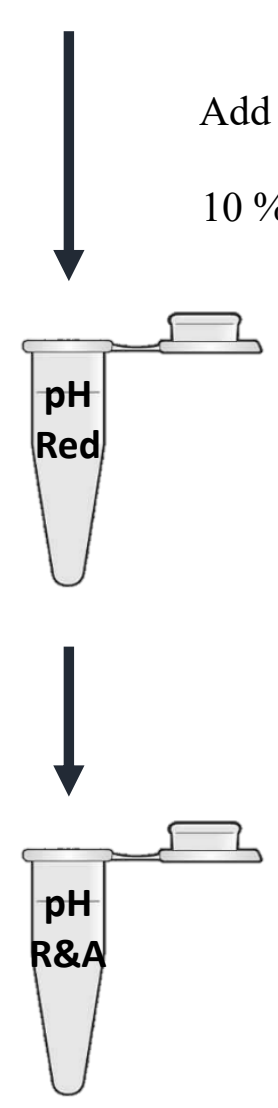

(7)

Add $500 \mathrm{mmol} / \mathrm{L}$ DTT to a final concentration of $5 \mathrm{mmol} / \mathrm{L}$;

Buffer composition in both vials is now

$10 \%$ Formulation, $89 \%$ Denaturing, $1 \% 500 \mathrm{mmol} / \mathrm{L}$ DTT;

Protein concentration is $1.0 \mu \mathrm{g} / \mu \mathrm{L}$

(8)

Denature/Reduce for 60 min at $4{ }^{\circ} \mathrm{C}$

(9)

Add $500 \mathrm{mmol} / \mathrm{L}$ IAM to final concentration of $10 \mathrm{mmol} / \mathrm{L}$<smiles>[18OH]</smiles>

Alkylate for $60 \mathrm{~min}$ at $4{ }^{\circ} \mathrm{C}$

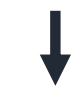

continued
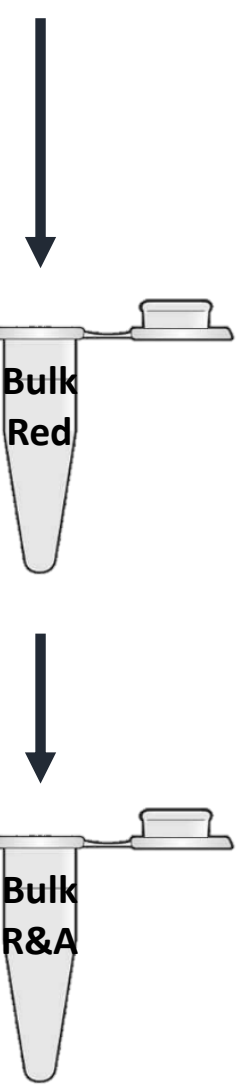
Figure S1 (continued). Preparation of pH Stress, Reference, Unknown and Spike Samples.

b) (continued) Tryptic digestion of pH stressed NISTmAb and unstressed NISTmAb.

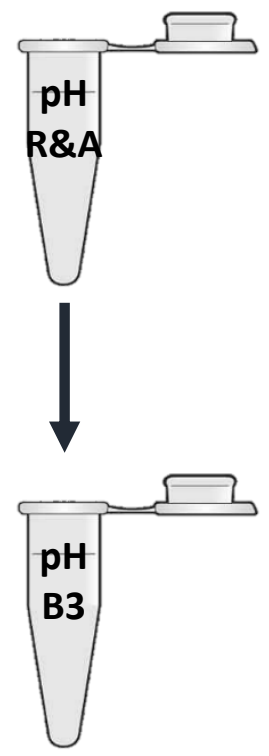

(from Step 10)

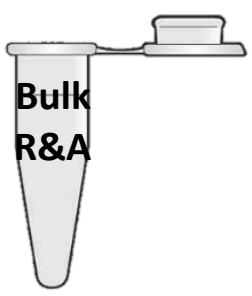

Exchange to Digestion Buffer (B3)
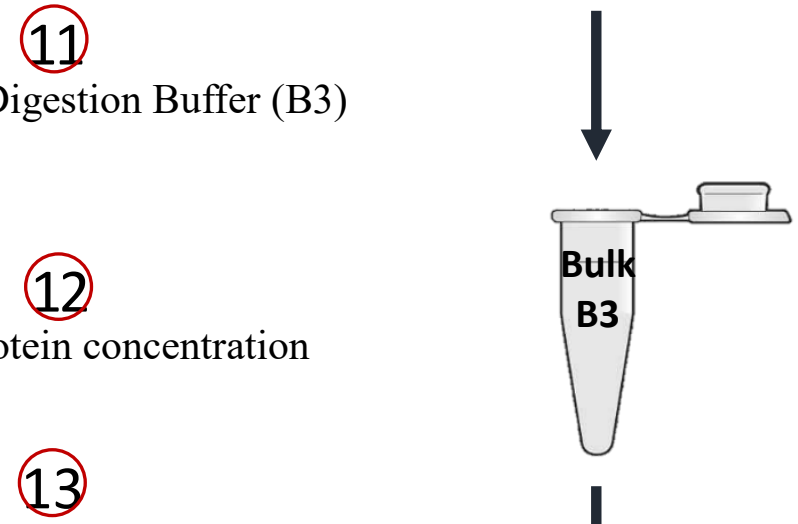

Transfer $x \mu \mathrm{g}$ protein; equalize both concentrations to $0.5 \mu \mathrm{g} / \mu \mathrm{L}$ with Digestion Buffer
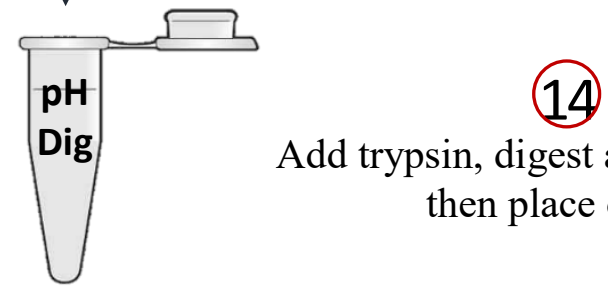

Add trypsin, digest at RT for 4 hrs, then place on ice

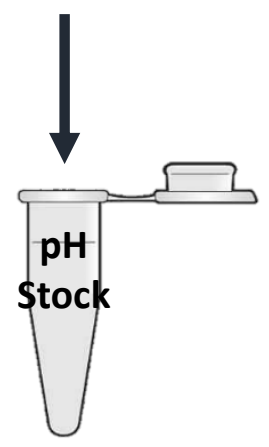

(15)

Transfer $x \mu \mathrm{g}$ protein
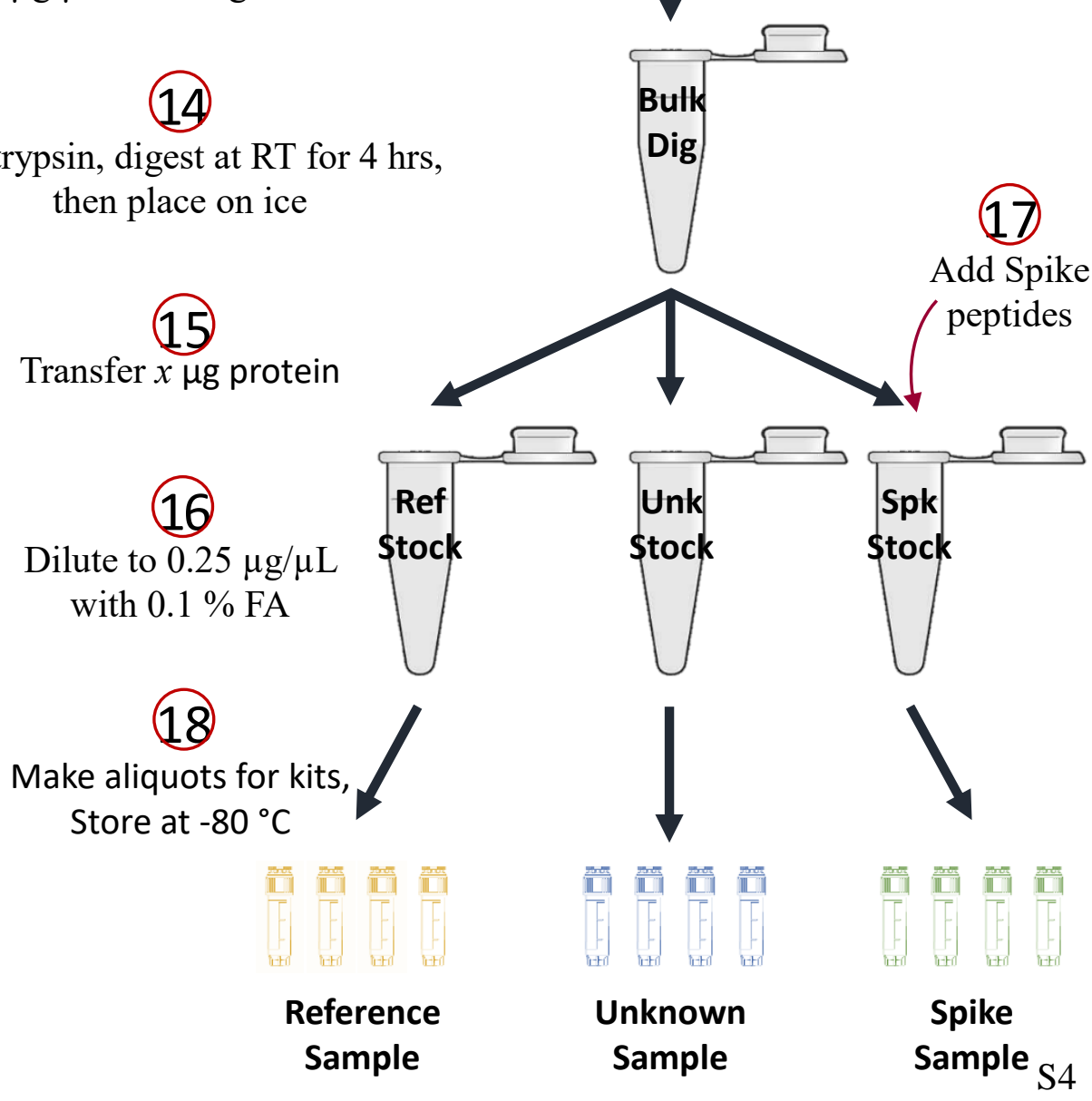

pH Stress

Sample 
a)

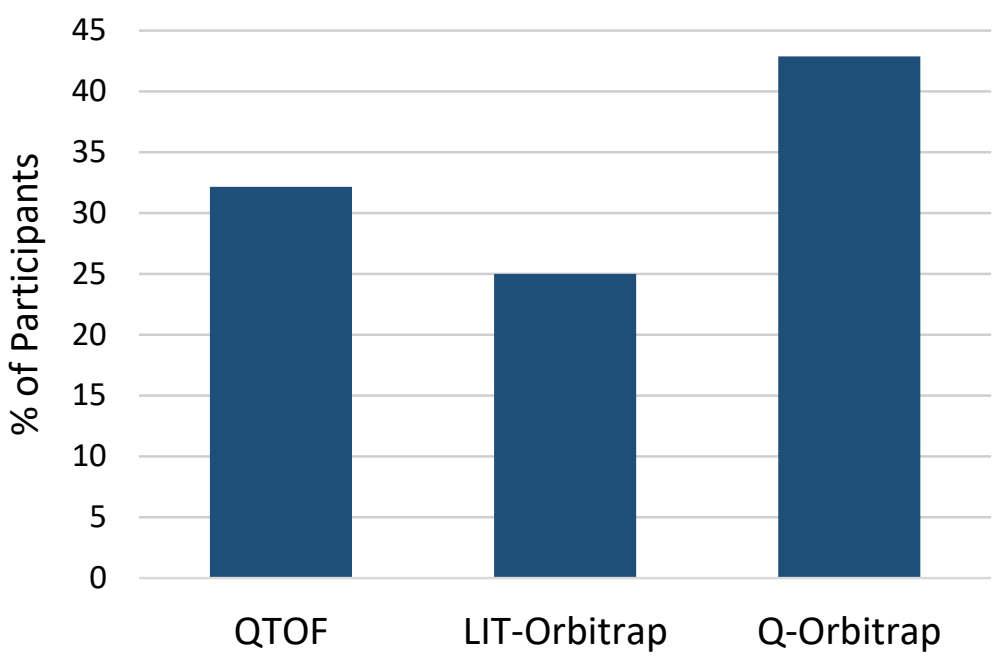

$n=28$ participants b)

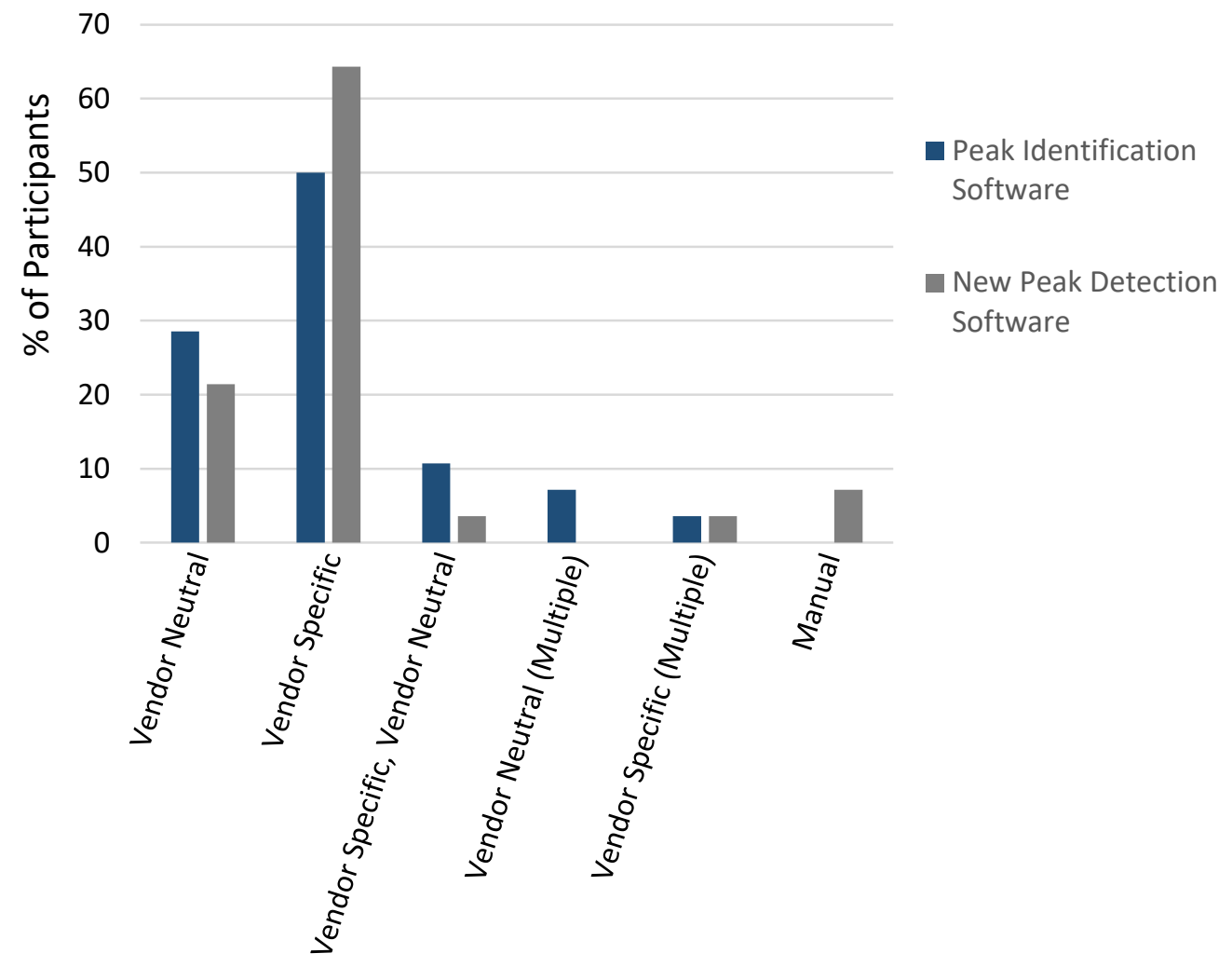

Figure S2. Survey of Instrument and Software Types Used for Data Acquisition and Analysis. a) Participants reported the mass spectrometer used to acquire data for the study. Instruments were then assigned to one of three general categories (QTOF, LIT-Orbitrap, or Q-Orbitrap) and the percentage of participants using each instrument type was calculated. b) Participants reported the software platforms used for peak identification and NPD. The platforms were then designated as vendor neutral, vendor specific or manual (NPD performed by visual inspection of the chromatogram) and the percentage of participants using each platform type or combination of types was calculated. 


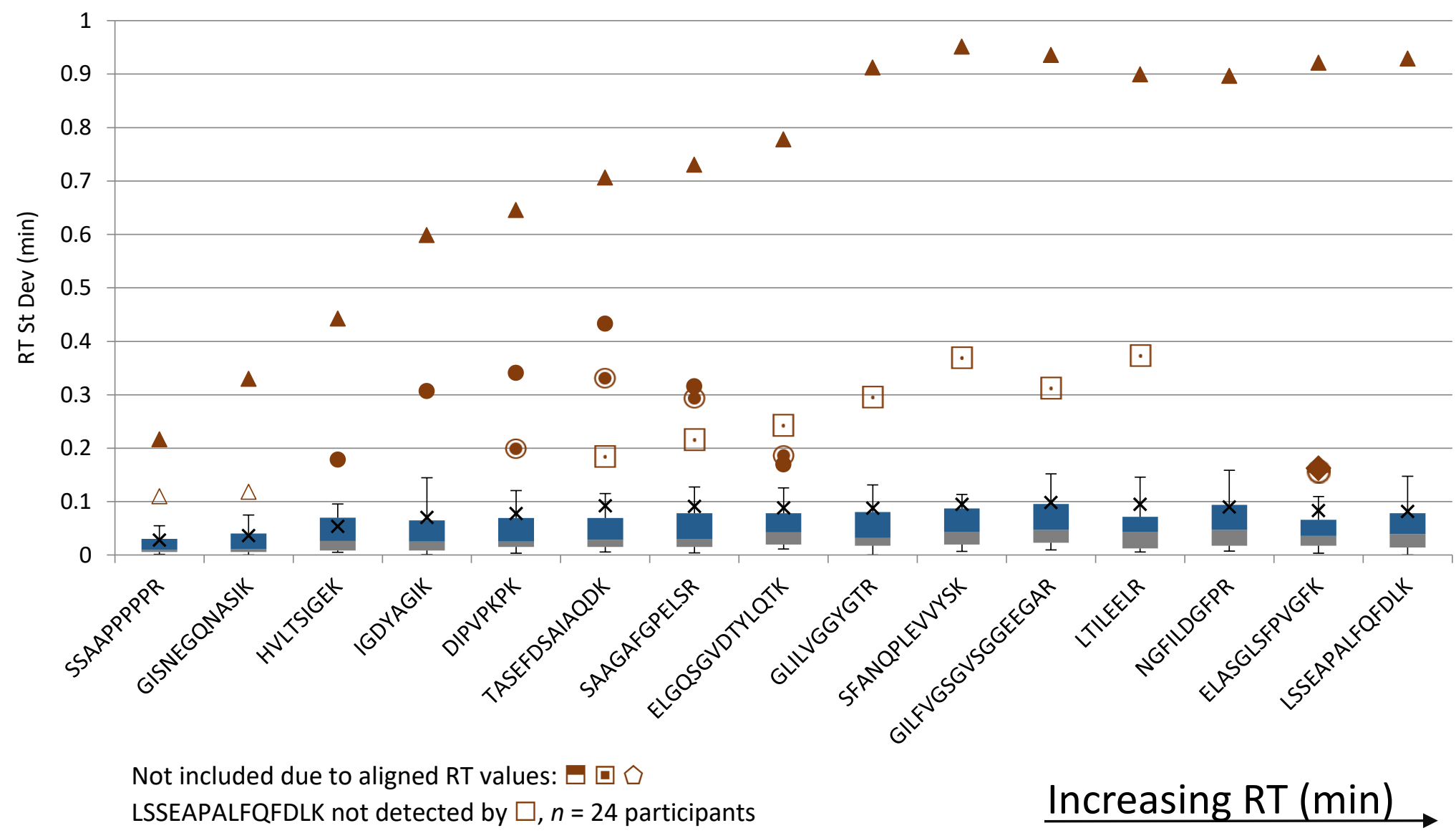

LSSEAPALFQFDLK not detected by $\square, n=24$ participants

$n=25$ participants for all other peptides

ELASGLSFPVGFK overlapping symbols: $\ominus$ and $\bullet$

Figure S3. Intralaboratory Standard Deviations of Calibration Sample Peptide Retention Times. For each participant the intralaboratory standard deviation (St Dev) of retention times (RT) $(s)$ across three injections of the Calibration Sample was calculated for each peptide. A box plot (see Supplemental Figure S16) was generated from these St Dev values. The peptides are listed in order of increasing retention time. Equation for $s$ is provided in Supplemental Appendix S3, Section A. 


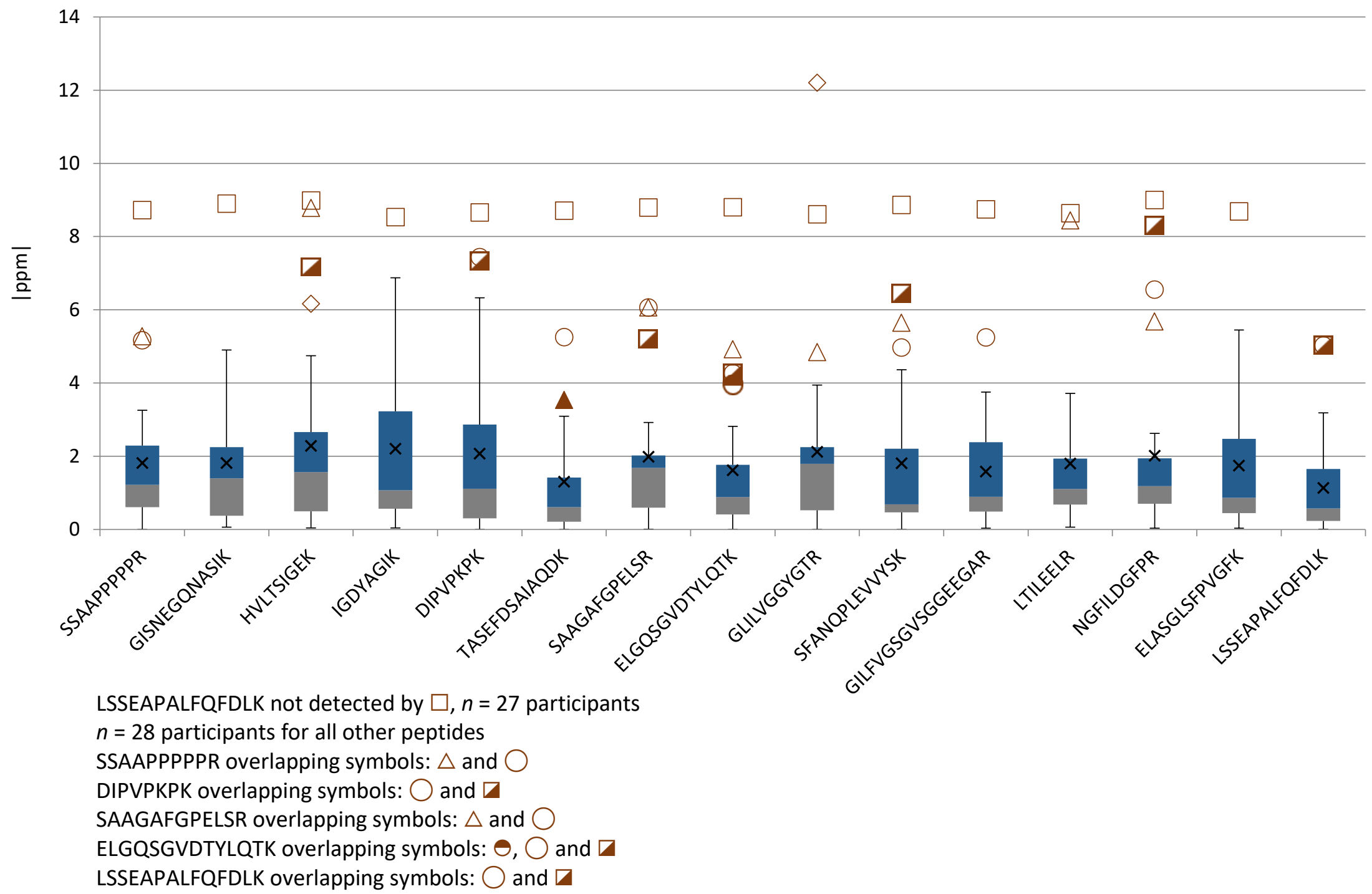

Figure S4. Intralaboratory Calibration Sample Peptide Mass Accuracy. The observed mass of each Calibration Sample peptide was reported by participants for one injection. Absolute ppm values of each peptide were then calculated between the observed and theoretical masses for each participant and used to generate a box plot (see Supplemental Figure S16). 


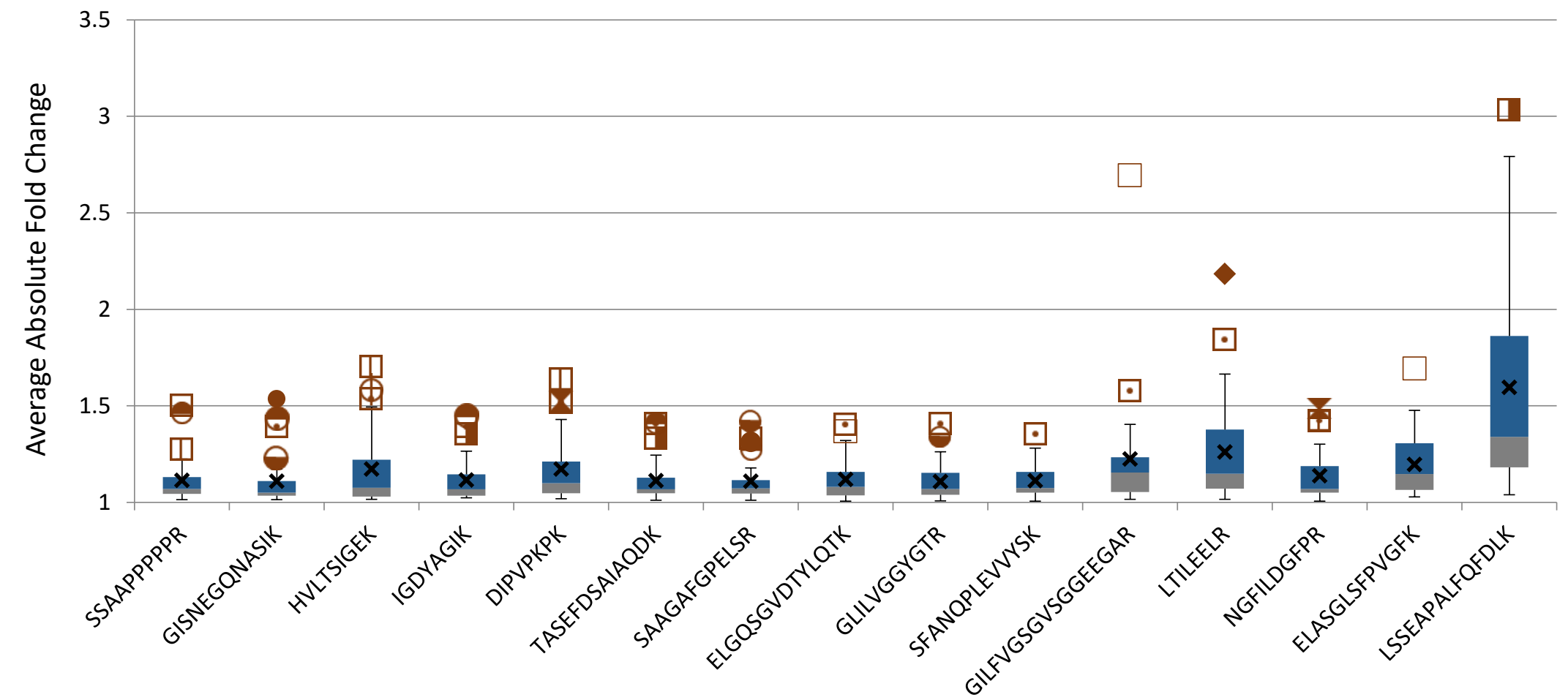

Values for $\square$ represent one absolute fold-change value rather than an average due to lack of third injection.

SSAAPPPPPR overlapping symbols: $\square$ and $\ominus$ IGDYAGIK overlapping symbols: $\ominus, \square$ and $\square$ DIPVPKPK overlapping symbols: $\mathbf{X}$ and $\square$ LSSEAPALFQFDLK not detected by $\square, n=27$ participants TASEFDSAIAQDK overlapping symbols: $\theta$ and $\square$ $n=28$ participants for all other peptides SAAGAFGPELSR overlapping symbols: $\ominus$, and $\odot$ ELGQSGVDTYLQTK overlapping symbols: $\square$ and $\square$ NGFILDGFPR overlapping symbols: $\square$ and $\square$

Figure S5. Intralaboratory Fold-Change Values of Calibration Sample Peptides. Absolute fold-change (FC) values were calculated from the summed XIC intensities provided by each participant across three injections (i.e. FC $1=$ injection 1 vs injection 2 ; FC $2=$ injection 1 vs injection 3; FC 3 = injection 2 vs injection 3, where the denominator in each FC ratio was determined by which of the two values was greater). Average values $(\bar{x})$ for the three injections were determined for each participant and used to generate a box plot for each peptide (see Supplemental Figure S16). Equation for $(\bar{x})$ is provided in Supplemental Appendix S3, Section A. 


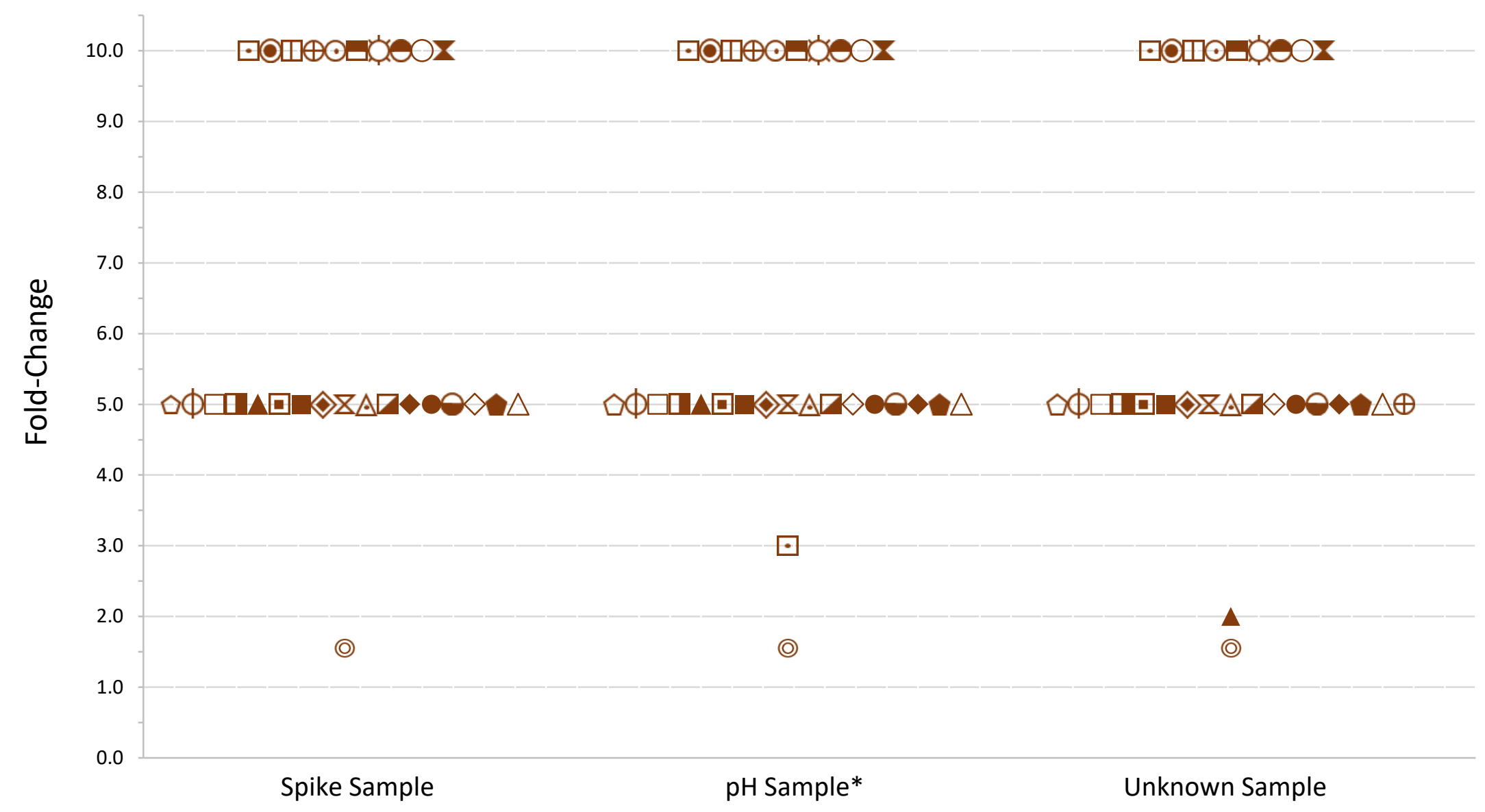

* Two fold-change thresholds were used by $\square:$ 3-fold for identified peaks and 10-fold for unidentified peaks.

Figure S6. Distribution of FCD Thresholds Applied. Participants reported the fold-change value used to define a change in peak abundance between each Test and Reference Sample. Each participant is represented by a unique symbol. 
a)

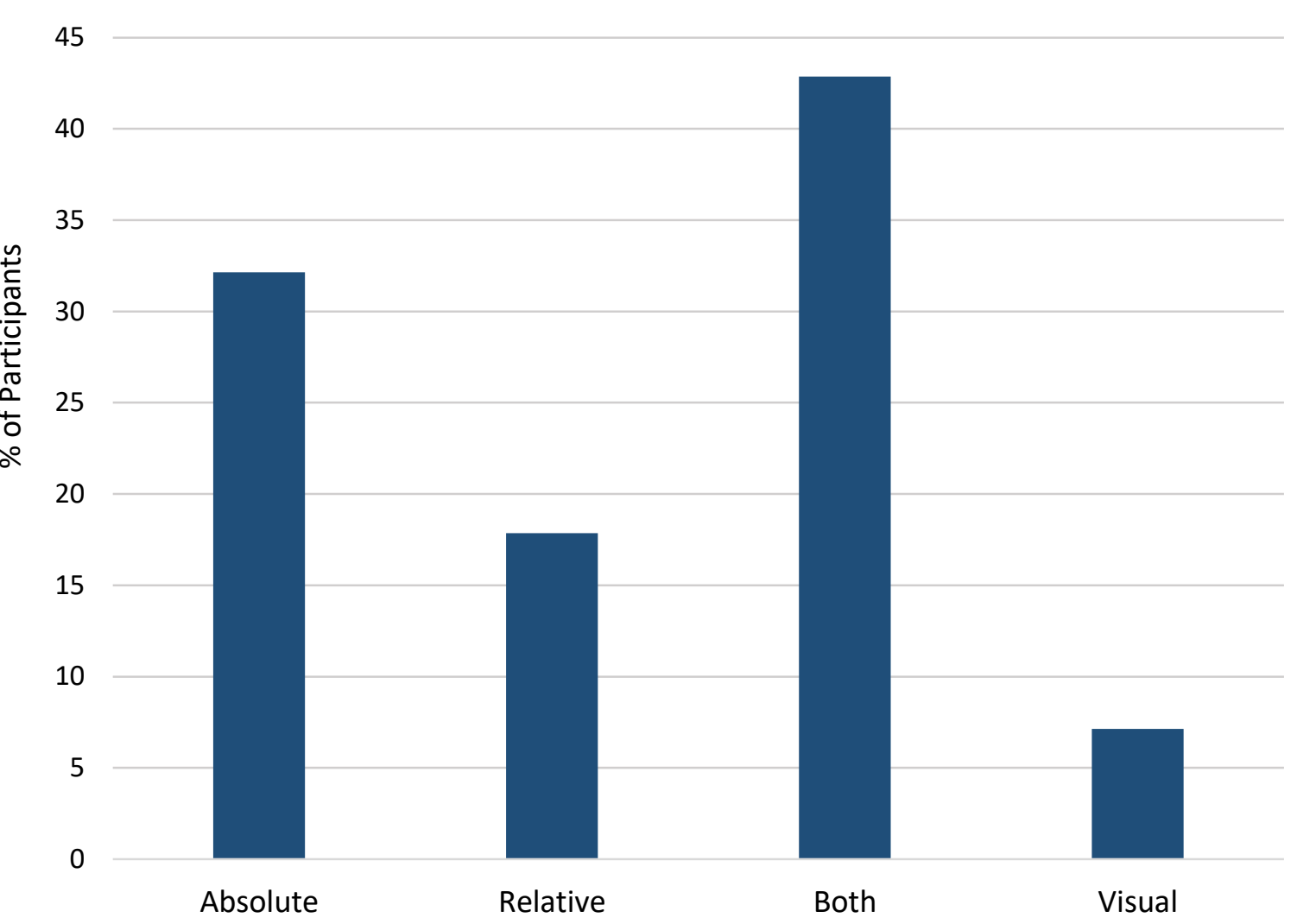

$n=28$ participants

Figure S7. Distribution of NPD Thresholds Applied. Participants reported a) the type of NPD threshold applied when performing NPD and the \% of participants utilizing each type (Absolute, Relative, Both Absolute and Relative, or Visual) was calculated; b) the value applied when using an absolute NPD threshold; and c) the value applied when using a relative NPD threshold. For b) and c) each participant is represented by a unique symbol. For b) the left panel shows the full set of data, the right panel is a zoomed view of data points $\leq 4.0 \mathrm{E} 4$. For $\mathrm{c}$ ) the top panel shows the full set of data, the bottom panel is a zoomed view of data points $\leq 0.3 \%$ 
b)

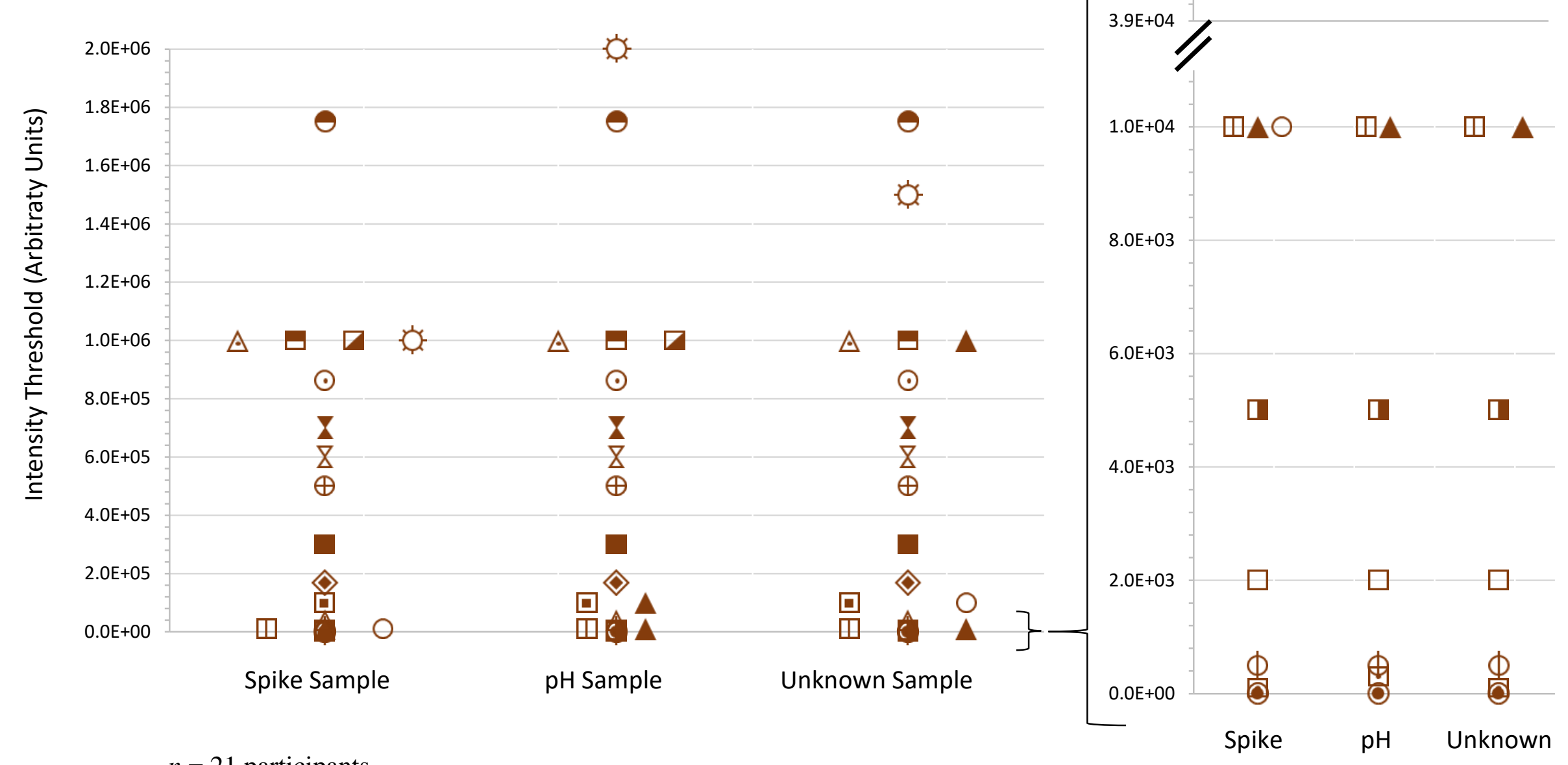

Figure S7 (continued). Distribution of NPD Thresholds Applied. Participants reported a) the type of NPD threshold applied when performing NPD and the \% of participants utilizing each type (Absolute, Relative, Both Absolute and Relative, or Visual) was calculated; b) the value applied when using an absolute NPD threshold; and c) the value applied when using a relative NPD threshold. For b) and c) each participant is represented by a unique symbol. For b) the left panel shows the full set of data, the right panel is a zoomed view of data points $\leq 4.0 \mathrm{E} 4$. For $\mathrm{c}$ ) the top panel shows the full set of data, the bottom panel is a zoomed view of data points $\leq 0.3 \%$. 
c)

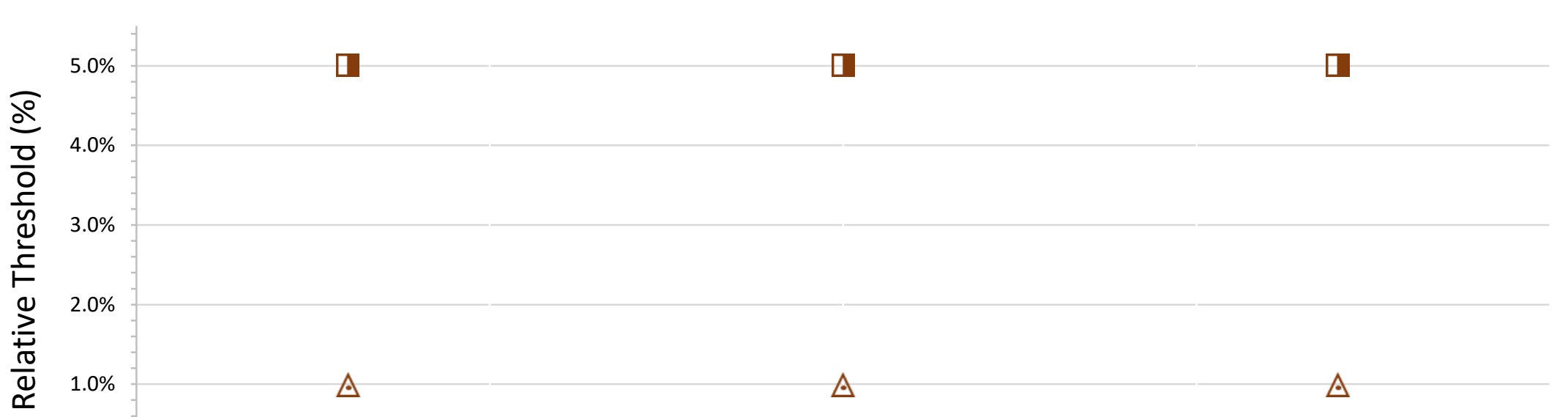

$0.0 \%$

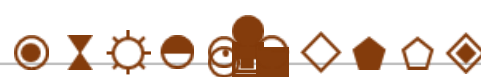

Spike Sample

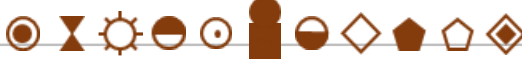

pH Sample
$0 \mathrm{x} \cdot \odot \odot$

Unknown Sample

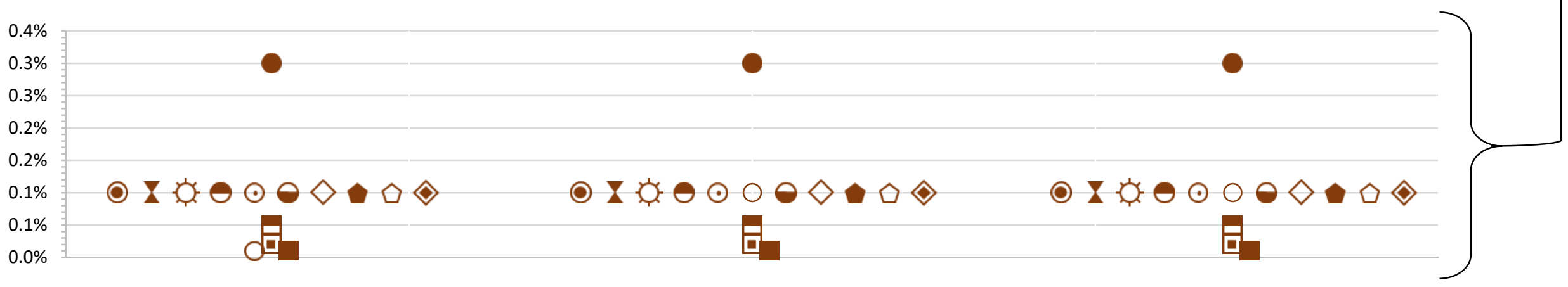

$n=17$ participants

Figure S7 (continued). Distribution of NPD Thresholds Applied. Participants reported a) the type of NPD threshold applied when performing NPD and the \% of participants utilizing each type (Absolute, Relative, Both Absolute and Relative, or Visual) was calculated; b) the value applied when using an absolute NPD threshold; and c) the value applied when using a relative NPD threshold. For b) and c) each participant is represented by a unique symbol. For b) the left panel shows the full set of data, the right panel is a zoomed view of data points $\leq 4.0 \mathrm{E} 4$. For $\mathrm{c}$ ) the top panel shows the full set of data, the bottom panel is a zoomed view of data points $\leq 0.3 \%$. 


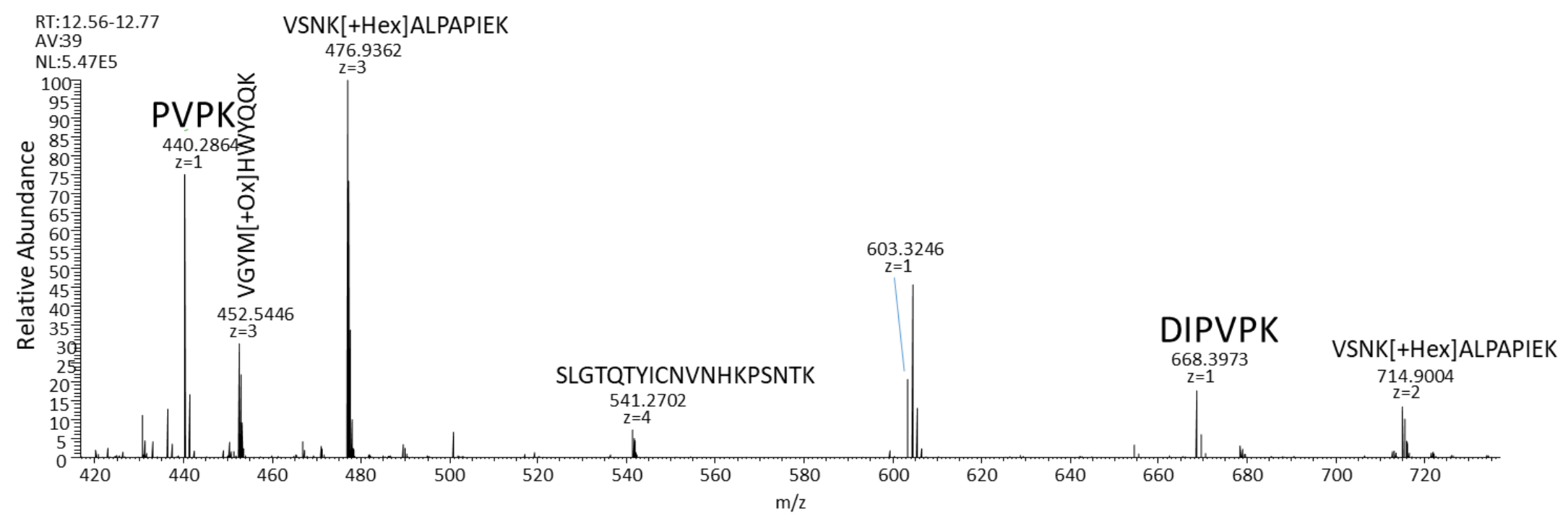

Figure S8. In-Source Fragment Spectra Generated by Spike Peptide DIPVPKPK. Spectra were summed across the extracted ion chromatogram peak of the DIPVPKPK fragments for Participant $\bullet$. Identified species are marked. If present, the doubly charge fulllength peptide would be found at $451.284 \mathrm{~m} / \mathrm{z}$. 


\section{0}

90

80

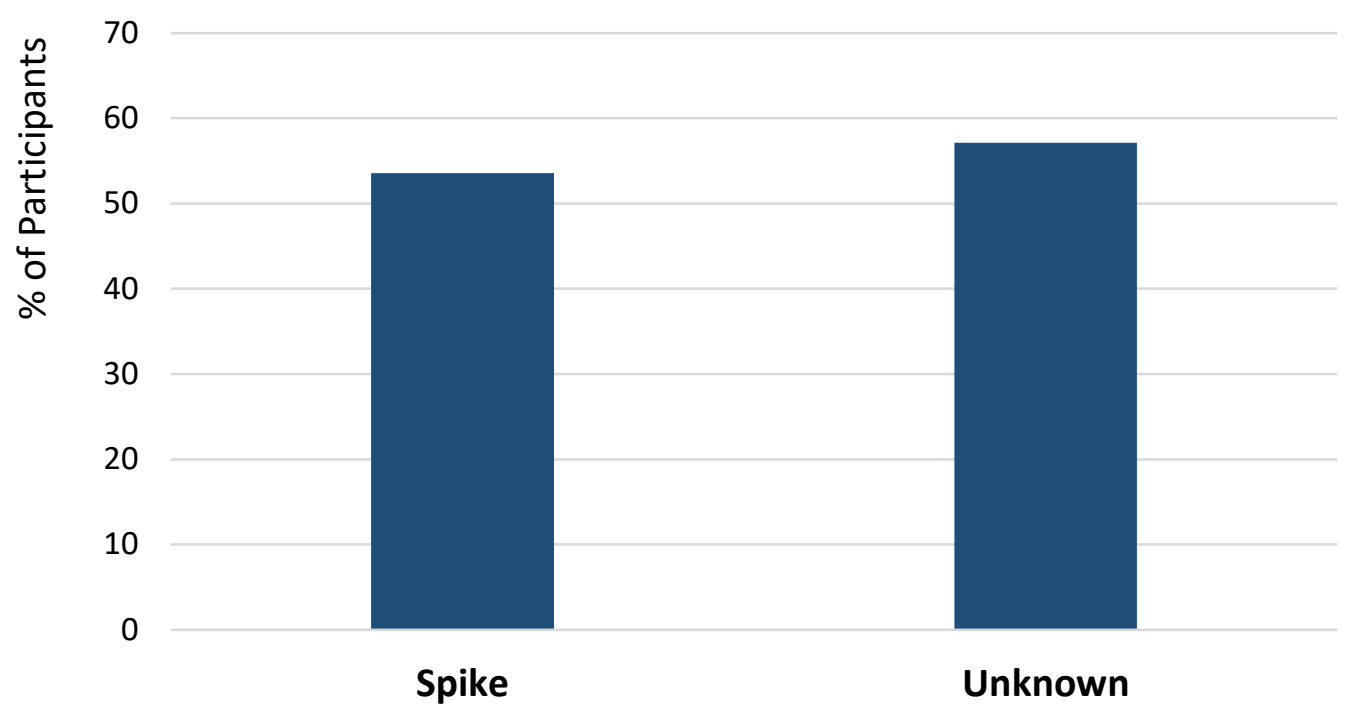

$n=28$ participants

Figure S9. Tally of Participants Whose Results Conformed to Expectation for Spike and Unknown NPD Analyses. Each participant's NPD results were surveyed to determine whether they conformed to the expected outcomes as described in the main text. Results are listed by individual participant in Figure 6. 
a)

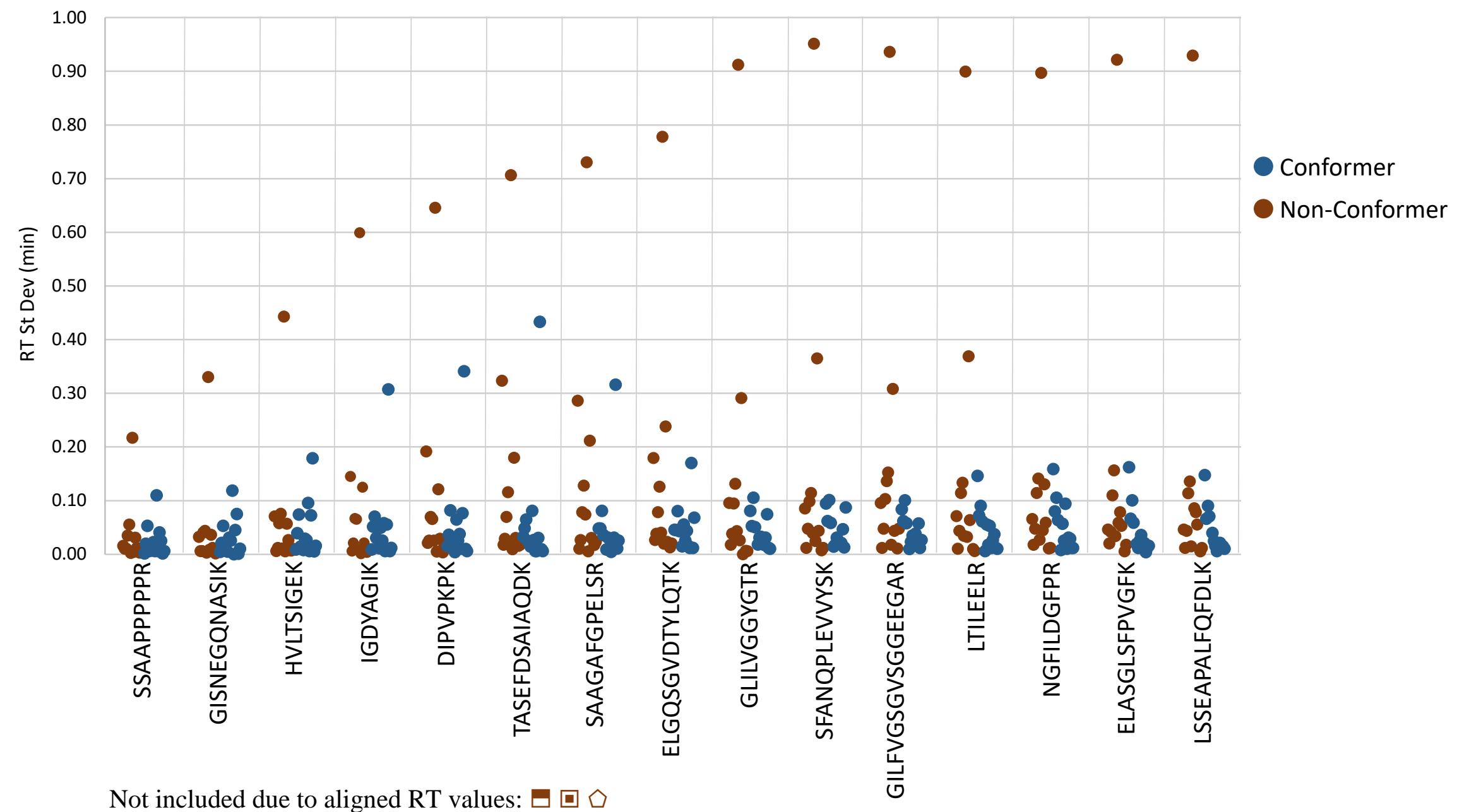

LSSEAPALFQFDLK not detected by $\square, n=24$ participants $n=25$ participants for all other peptides

Figure S10. Instrument Performance Relative to Conformity Status. Intralaboratory instrument performance values for a) retention time standard deviation, b) $|\mathrm{ppm}|$, c) absolute fold-change and d) absolute fold-change standard deviation are shown for each Calibration Peptide. Participants whose NPD results conformed to expectation for both Spike and Unknown Samples are shown in blue; those who did not conform are shown in red. 
b)

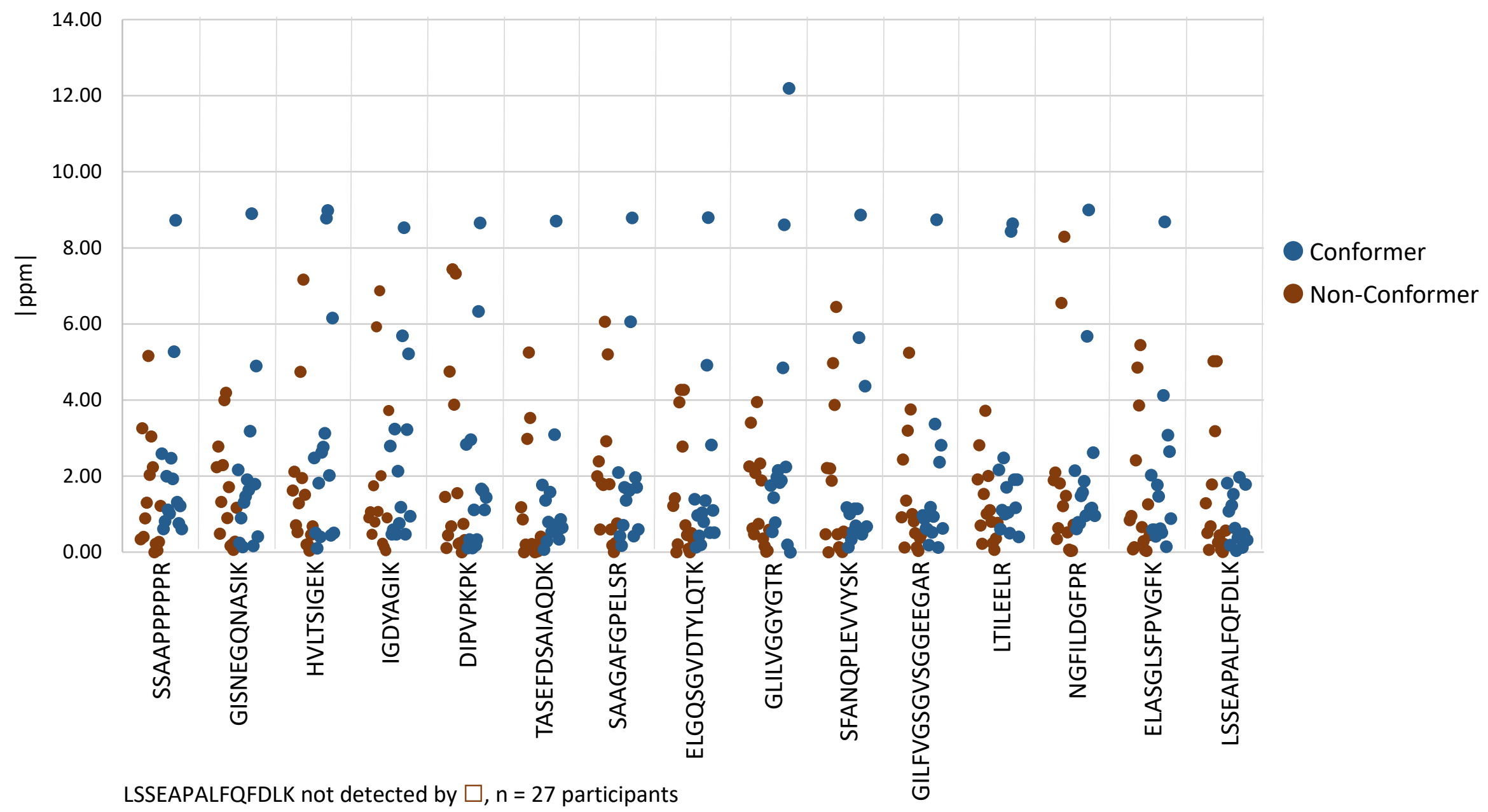

$\mathrm{n}=28$ participants for all other peptides

Figure S10 (continued). Instrument Performance Relative to Conformity Status. Intralaboratory instrument performance values for a) retention time standard deviation, b) $|\mathrm{ppm}|$, c) absolute fold-change and d) absolute fold-change standard deviation are shown for each Calibration Peptide. Participants whose NPD results conformed to expectation for both Spike and Unknown Samples are shown in blue; those who did not conform are shown in red. 
C)

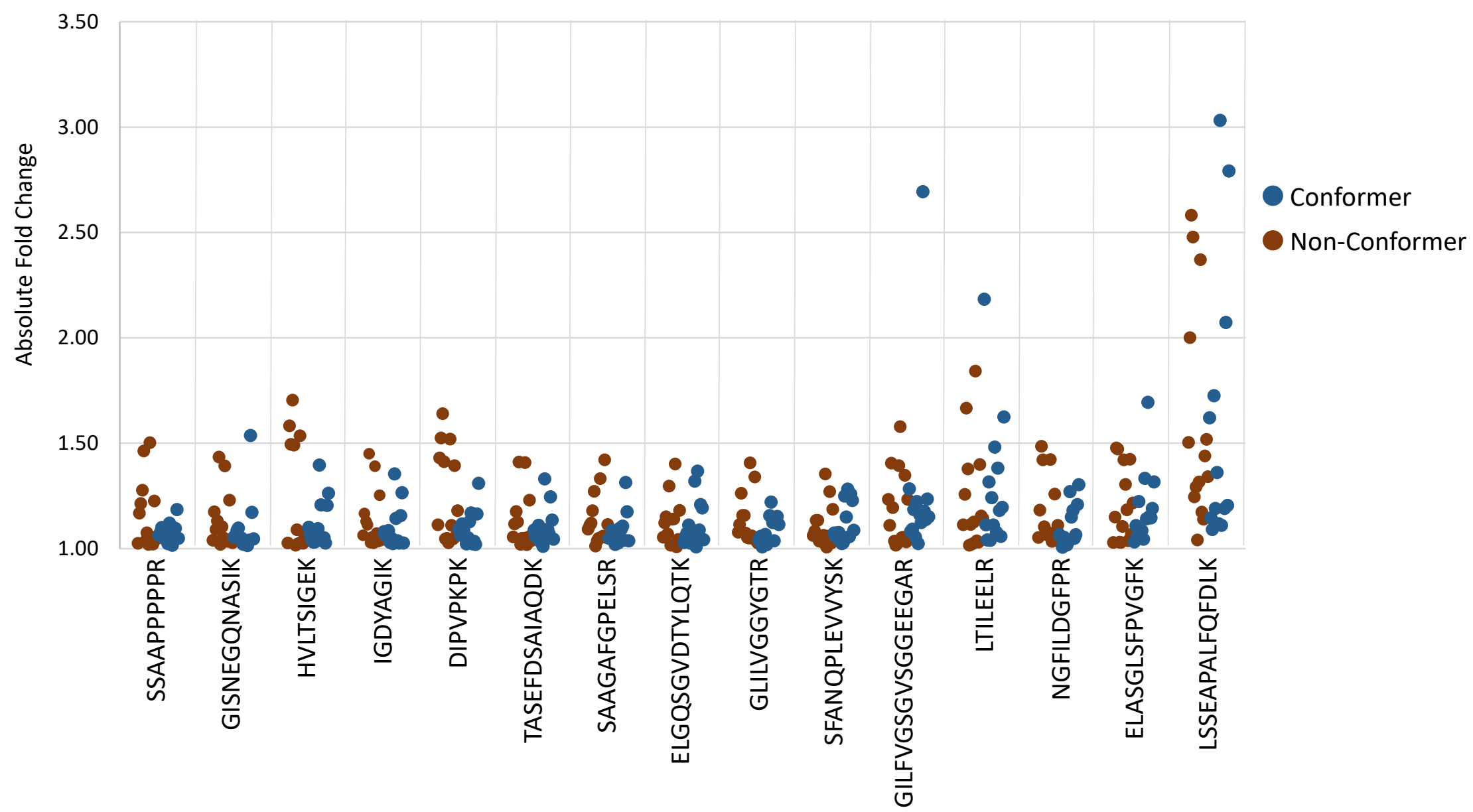

Values for $\square$ represent one absolute fold-change value rather than an average due to lack of third injection.

LSSEAPALFQFDLK not detected by $\square, n=27$ participants

$n=28$ participants for all other peptides

Figure S10 (continued). Instrument Performance Relative to Conformity Status. Intralaboratory instrument performance values for a) retention time standard deviation, b) $|\mathrm{ppm}|$, c) absolute fold-change and d) absolute fold-change standard deviation are shown for each Calibration Peptide. Participants whose NPD results conformed to expectation for both Spike and Unknown Samples are shown in blue; those who did not conform are shown in red. 
d)

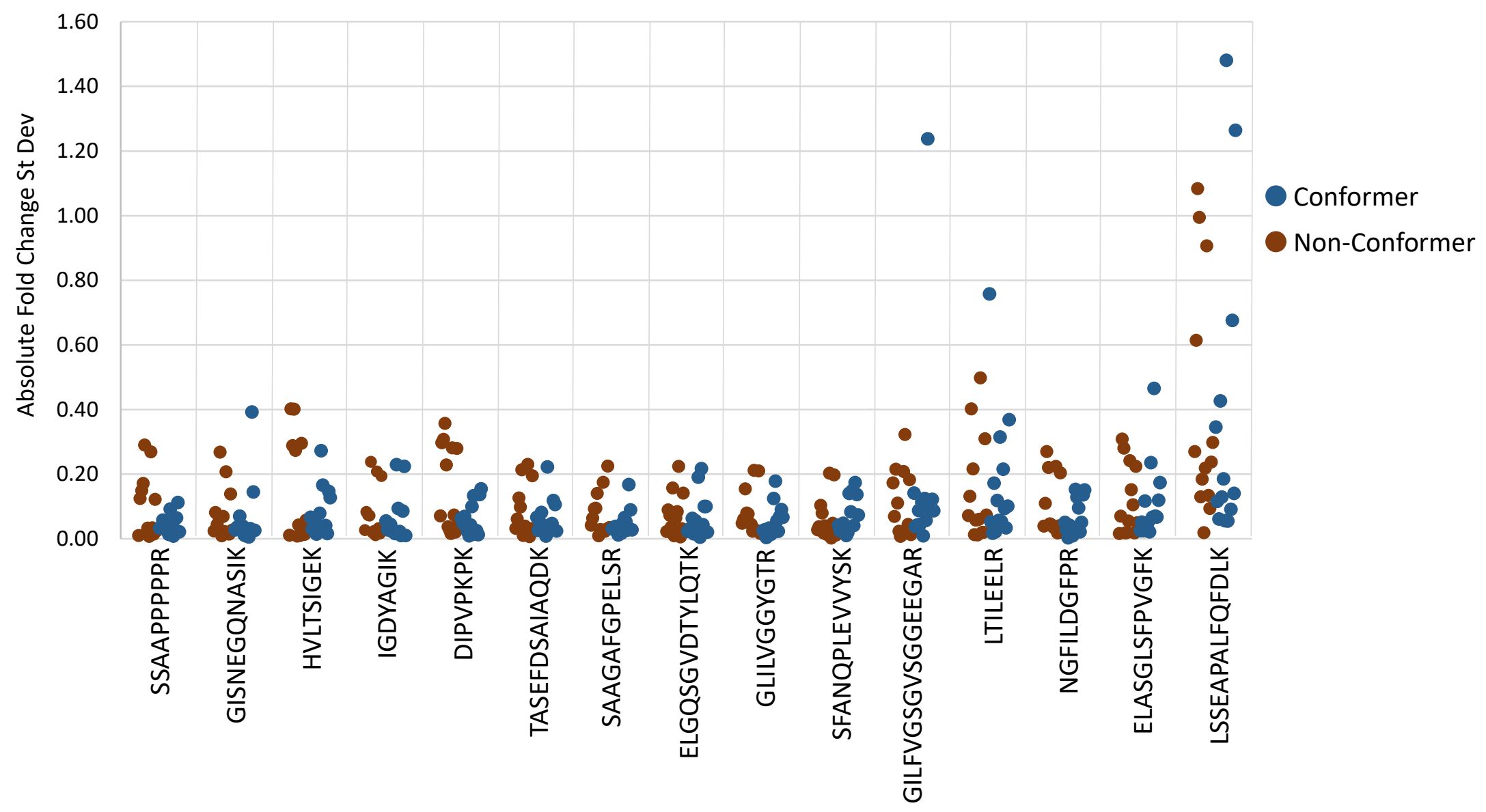

Values for $\triangle$ represent one absolute fold-change value rather than an average due to lack of third injection.

LSSEAPALFQFDLK not detected by $\square, n=27$ participants

$n=28$ participants for all other peptides

Figure S10 (continued). Instrument Performance Relative to Conformity Status. Intralaboratory instrument performance values for a) retention time standard deviation, b) $\mathrm{ppm} \mid$, c) absolute fold-change and d) absolute fold-change standard deviation are shown for each Calibration Peptide. Participants whose NPD results conformed to expectation for both Spike and Unknown Samples are shown in blue; those who did not conform are shown in red. 
a)

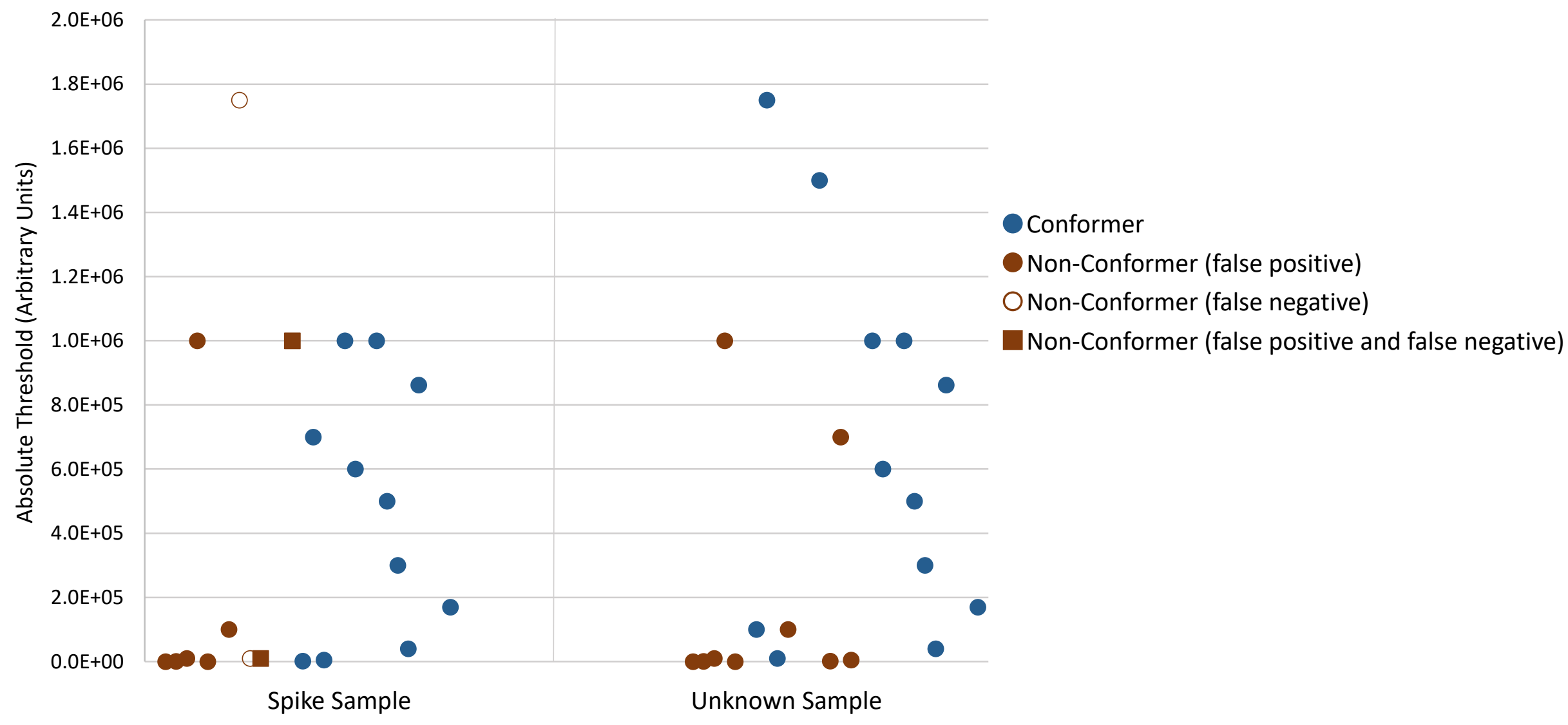

$n=21$ participants

Figure S11. NPD Thresholds Relative to Conformity Status. Individual participant's NPD parameters for a) absolute NPD threshold, and b) relative NPD threshold are shown for the Spike and Unknown Samples, with colors and shading indicating conformity status for that sample only. 
b)

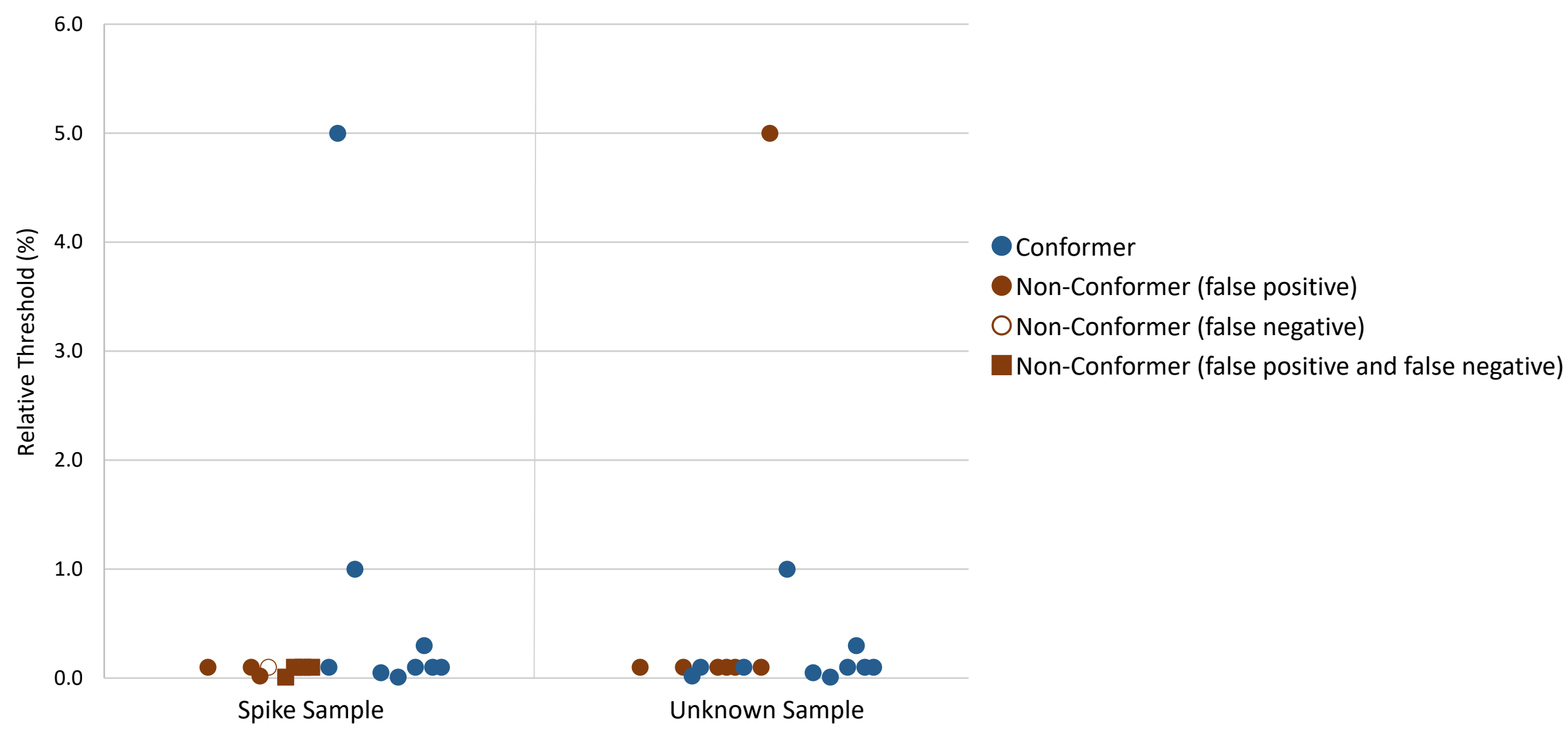

$n=17$ participants

Figure S11 (continued). NPD Thresholds Relative to Conformity Status. Individual participant's NPD parameters for a) absolute NPD threshold, and b) relative NPD threshold are shown for the Spike and Unknown Samples, with colors and shading indicating conformity status for that sample only. 


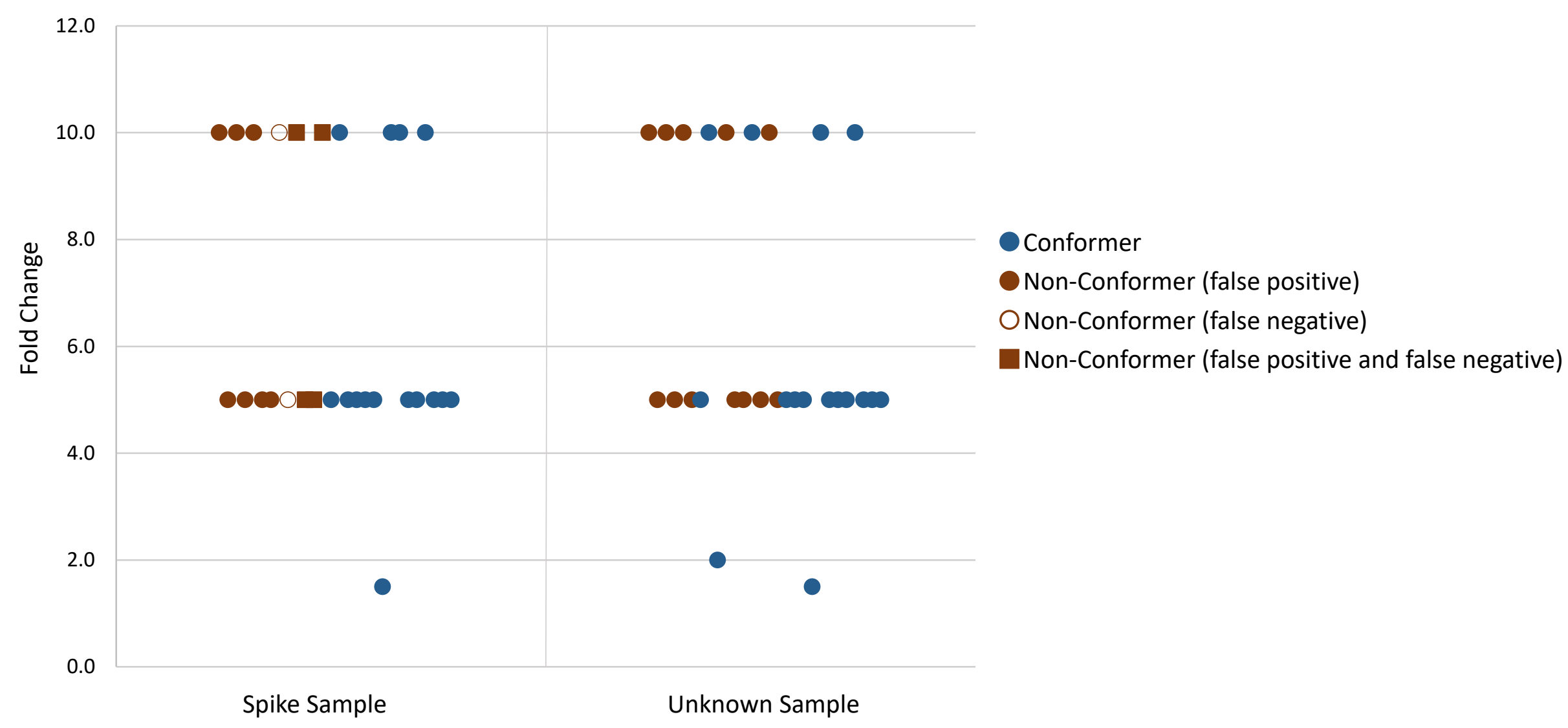

Figure S12. FCD Thresholds Relative to Conformity Status. Individual participant's FCD thresholds used to indicate a change in abundance between each Test and Reference Sample are shown for the Spike and Unknown Samples, with colors and shading indicating conformity status for that sample only. 
25

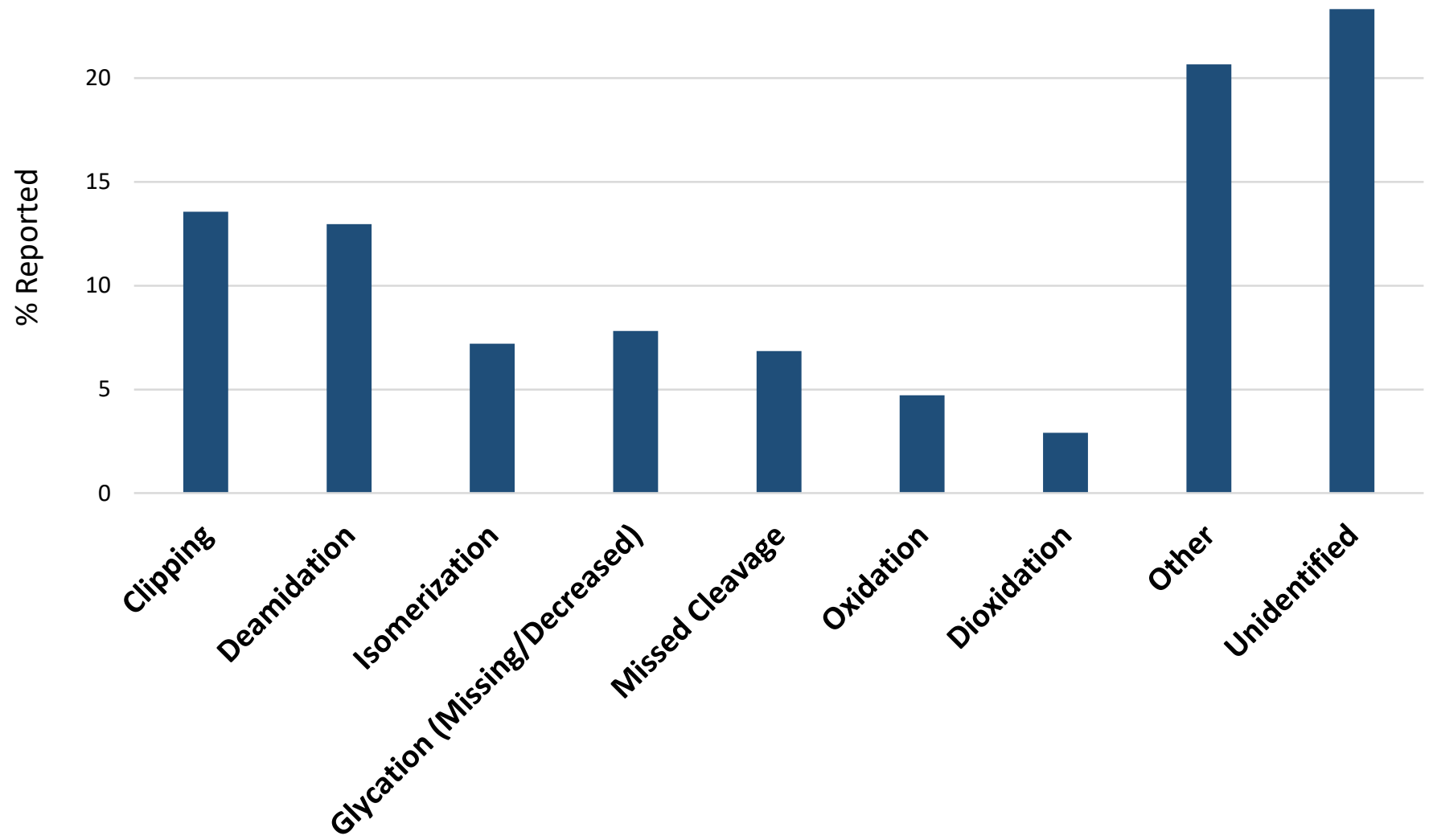

Figure S13. Survey of Modifications Reported in pH Stress Sample. Participants performed NPD comparing the pH Stress Sample to the Reference Sample and reported peaks that were considered new, missing, or changed in abundance between pH Stress and Reference Samples. The frequency with which various modifications were reported as new, missing, or changed is summarized here, highlighting the seven most commonly reported modifications. The \% Reported is calculated as: $\frac{\text { Sall peptides reported with given modification }}{\text { Eall reported peaks }} 100$, where the peptides are summed across all participants. When multiple modifications occurred on the same peptide, each modification was counted separately. The tally of missed cleavage peptides includes only unmodified peptides. Peptides with in-source modifications are not included. The sum of peaks in the denominator includes identified and unidentified species. See Supplemental Table S8 for a full list of reported peaks. 


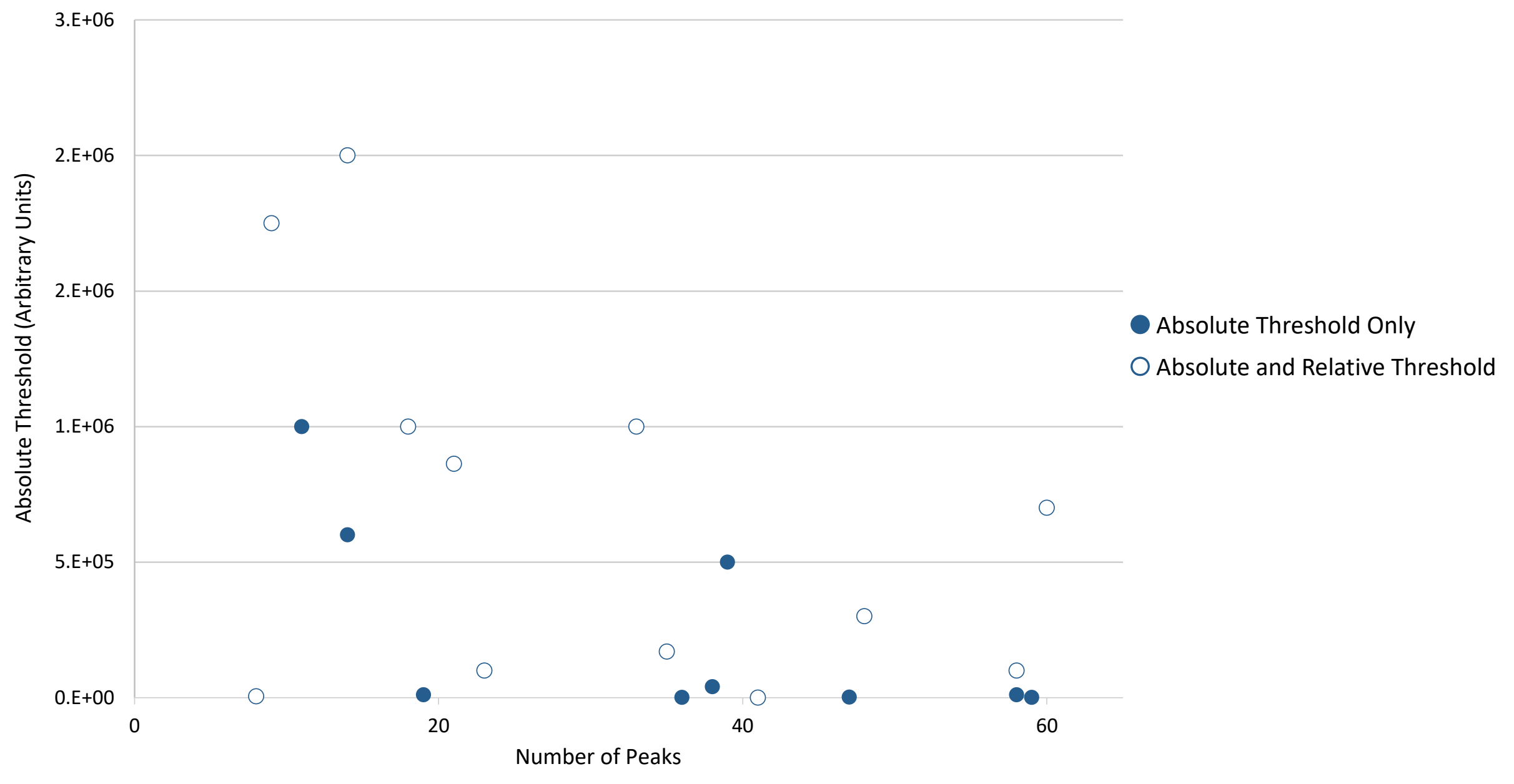

Figure S14. Number of Peaks Reported in pH Stress Sample Compared to Absolute NPD threshold Applied. The number of peaks reported as new, missing, or changed in abundance in the pH Stress Sample by each participant who utilized an absolute NPD threshold to determine true peak single during NPD analysis is plotted against the absolute NPD threshold value. Participants who only used an absolute NPD threshold to define true peaks are distinguished from those who used a relative NPD threshold in addition to the absolute NPD threshold to define peaks. 
b)

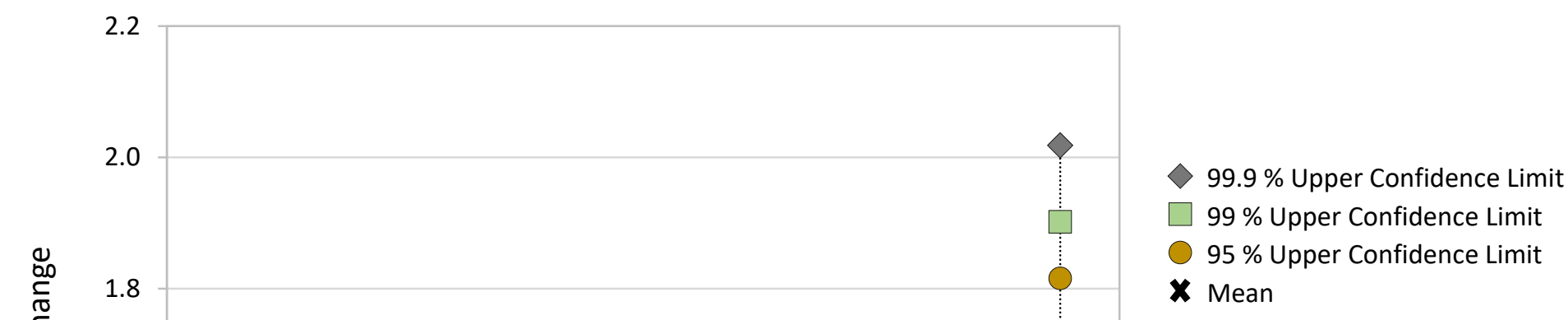

Figure S15 (continued). Potential Methods for Determining FCD Threshold. For each Calibration Peptide the mean absolute foldchange was calculated across all participants along with a) reproducibility standard deviation $\left(s_{\mathrm{R}}\right)$; b) upper confidence limits; and c) upper tolerance limits. Panel (a) shows each peptide mean plus $2\left(s_{\mathrm{R}}\right)$ and each peptide mean plus $3\left(s_{\mathrm{R}}\right)$. Panel (b) shows each peptide mean and its $99.9 \%$ upper confidence limit, $99 \%$ upper confidence limit and $95 \%$ upper confidence limit. Panel (c) shows each peptide mean and upper tolerance limits with $99 \%$ confidence/ $99 \%$ coverage; $95 \%$ confidence/ $99 \%$ coverage; $99 \%$ confidence/ $95 \%$ coverage; and $95 \%$ confidence/ $95 \%$ coverage. Note that for this dataset the confidence limits and tolerance limits shown in Panels (b) and (c) are based on the lognormal distribution. Note that for DIPVPKPK, in Panel (c) the 3-parameter lognormal maximum likelihood estimation did not converge and thus no tolerance limits are shown for this peptide. 
c)

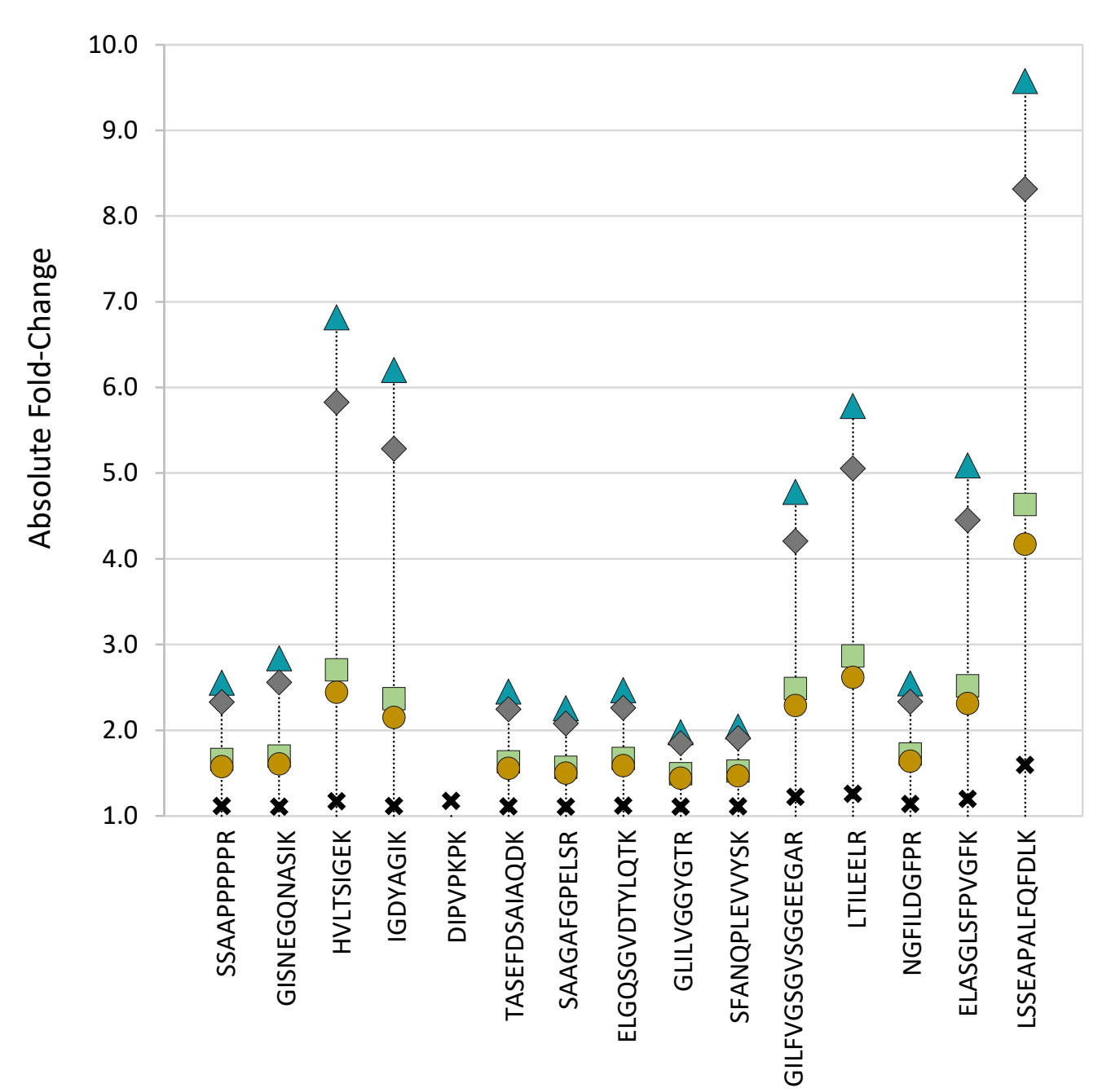

$\triangle 99 \%$ Confidence; $99 \%$ Coverage $\checkmark 5 \%$ Confidence; $99 \%$ Coverage $\square 99 \%$ Confidence; $95 \%$ Coverage $95 \%$ Confidence; $95 \%$ Coverage $\mathbf{x}$ Mean

Figure S15 (continued). Potential Methods for Determining FCD Threshold. For each Calibration Peptide the mean absolute foldchange was calculated across all participants along with a) reproducibility standard deviation $\left(s_{\mathrm{R}}\right)$; b) upper confidence limits; and c) upper tolerance limits. Panel (a) shows each peptide mean plus $2\left(s_{R}\right)$ and each peptide mean plus $3\left(s_{R}\right)$. Panel (b) shows each peptide mean and its $99.9 \%$ upper confidence limit, $99 \%$ upper confidence limit and $95 \%$ upper confidence limit. Panel (c) shows each peptide mean and upper tolerance limits with $99 \%$ confidence/ $99 \%$ coverage; $95 \%$ confidence/ $99 \%$ coverage; $99 \%$ confidence/ $95 \%$ coverage; and $95 \%$ confidence/ $95 \%$ coverage. Note that for this dataset the confidence limits and tolerance limits shown in Panels (b) and (c) are based on the lognormal distribution. Note that for DIPVPKPK, in Panel (c) the 3-parameter lognormal maximum likelihood estimation did not converge and thus no tolerance limits are shown for this peptide. 
Outlier: Greater than the upper quartile by more than $1.5 x$ the interquartile range, unique symbol for each participant
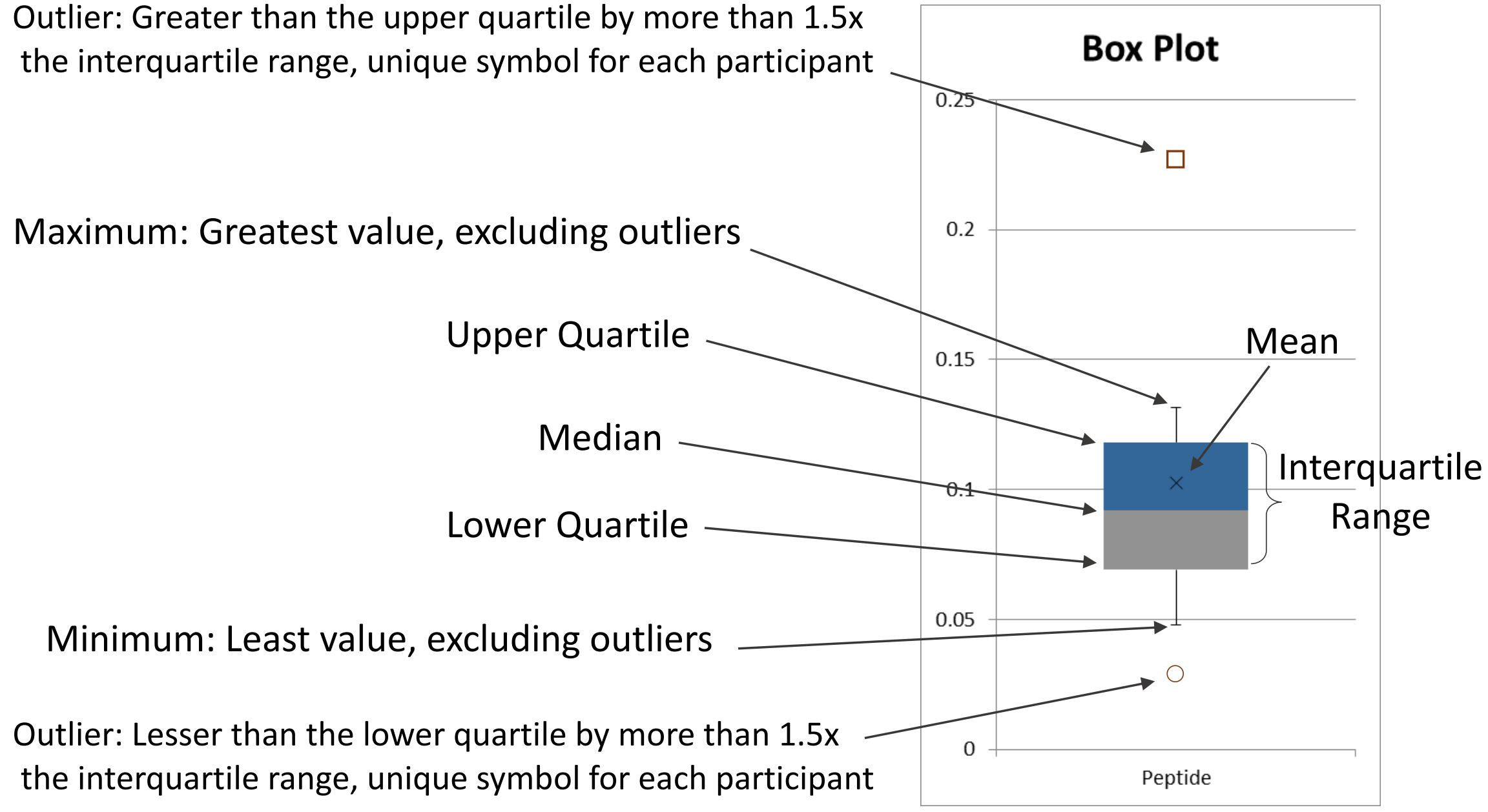

Figure S16. Summary of Box Plot Interpretation. Each component of the box plot graphs used throughout this manuscript is described. The Upper Quartile represents the median of data values that lie above the Median of all data values, while the Lower Quartile represents the median of data values that fall below the Median of all data values. Unique outlier symbols represent the same participant throughout all figures. The mean, median, upper and lower quartile values include outlier values. Outliers are only excluded when calculating maximum and minimum values. 


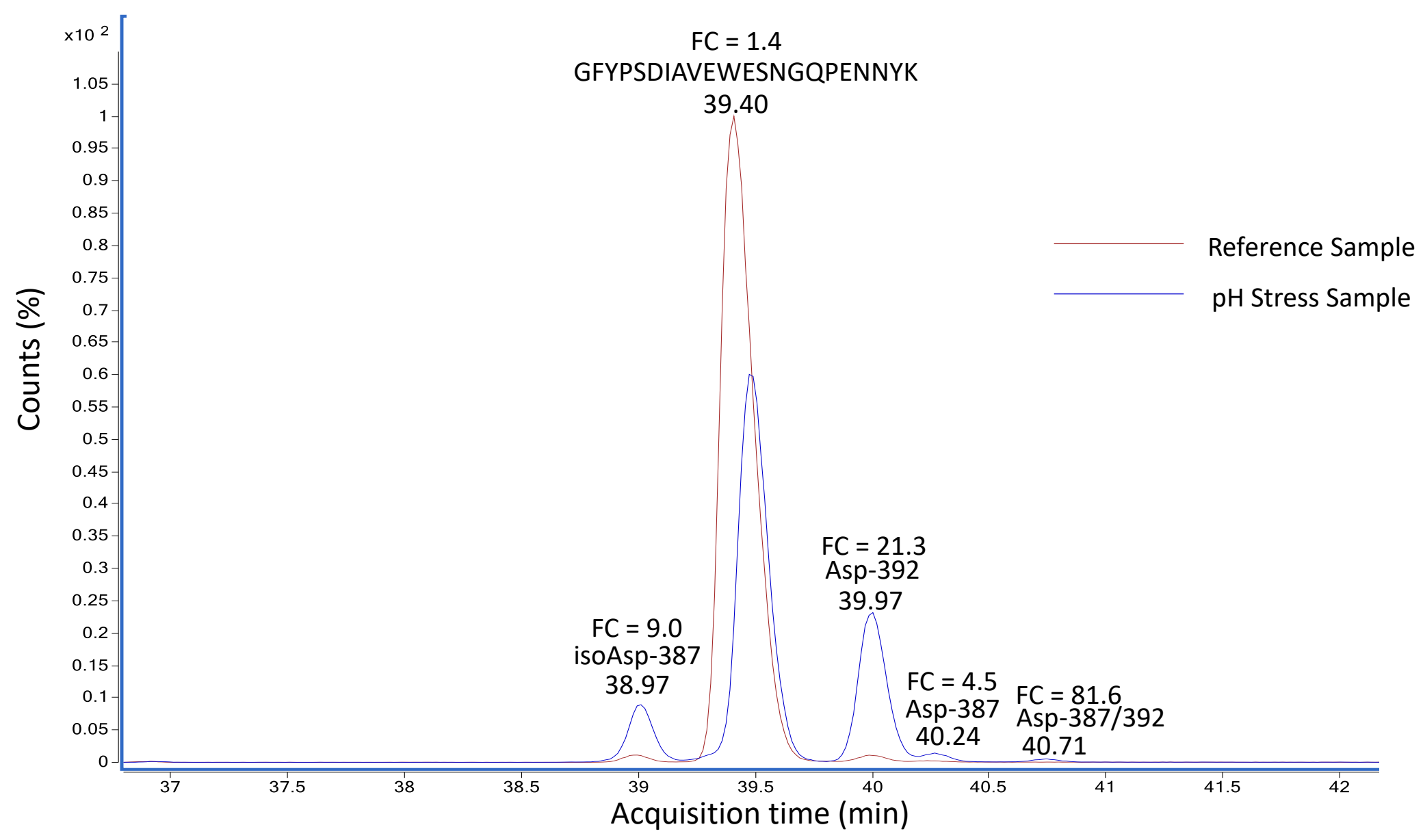

Figure S17. Extracted Ion Chromatograms of PENNY Peptide Deamidation Species. Raw data generated by Participant for the Reference and $\mathrm{pH}$ Stress Samples were reviewed for detection of GFYPSDIAVEWESNGQPENNYK deamidation species (heavy chain residues 374-395). Peaks corresponding to the extracted ion chromatograms for the unmodified peptide, three singly deamidated peptides and one doubly deamidated peptide are shown. The red trace represents the Reference Sample, while the blue trace represents the $\mathrm{pH}$ Stress Sample. Peaks are labeled with the fold-change value between corresponding $\mathrm{pH}$ Stress and Reference Sample peaks (as determined by the participant's NPD processing platform), the site of the deamidation, and the retention time. 